\title{
LINEAR INDEPENDENCE OF BOUNDARY TRACES OF EIGENFUNCTIONS OF ELLIPTIC AND STOKES OPERATORS AND APPLICATIONS
}

Abstract. This paper is divided into two parts and focuses on the linear independence of boundary traces of eigenfunctions of boundary value problems. Part I deals with second-order elliptic operators, and Part II with Stokes (and Oseen) operators.

Part I: Let $\lambda_{i}$ be an eigenvalue of a second-order elliptic operator defined on an open, sufficiently smooth, bounded domain $\Omega$ in $\mathbb{R}^{n}$, with Neumann homogeneous boundary conditions on $\Gamma=\partial \Omega$. Let $\left\{\varphi_{i j}\right\}_{j=1}^{\ell_{i}}$ be the corresponding linearly independent (normalized) eigenfunctions in $L_{2}(\Omega)$, so that $\ell_{i}$ is the geometric multiplicity of $\lambda_{i}$. We prove that the Dirichlet boundary traces $\left\{\left.\varphi_{i j}\right|_{\Gamma_{1}}\right\}_{j=1}^{\ell_{i}}$ are linearly independent in $L_{2}\left(\Gamma_{1}\right)$. Here $\Gamma_{1}$ is an arbitrary open, connected portion of $\Gamma$, of positive surface measure. The same conclusion holds true if the setting \{Neumann B.C., Dirichlet boundary traces\} is replaced by the setting \{Dirichlet B.C., Neumann boundary traces $\}$. These results are motivated by boundary feedback stabilization problems for parabolic equations [L-T.2].

Part II: The same problem is posed for the Stokes operator with motivation coming from the boundary stabilization problems in [B-L-T.1][B-L-T.3] (with tangential boundary control), and [R] (with just boundary control), where we take $\Gamma_{1}=\Gamma$.

The aforementioned property of boundary traces of eigenfunctions critically hinges on a unique continuation result from the boundary of corresponding over-determined problems. This is well known in the case of second-order elliptic operators of Part I; but needs to be established in the case of Stokes operators. A few proofs are given here.

2000 Mathematics Subject Classification: 35L20, 47, 49K20, 76N25, 76Q05, 93B29, $93 \mathrm{C} 20$.

Key words and phrases: boundary traces of eigenfunctions, elliptic and Stokes operators. 


\section{PART I: SECOND-ORDER ELLIPTIC OPERATORS}

\section{I.1. Problem setting. Linear independence of boundary traces} of eigenfunctions. This part deals with the following question, which we formulate in the simplest canonical setting: Given an eigenvalue $\lambda_{i}$ of the Dirichlet Laplacian (respectively, of the Neumann Laplacian) defined on a suitably smooth bounded domain $\Omega$ in $\mathbb{R}^{n}$, with geometric multiplicity $\ell_{i}$, are the $\ell_{i}$ Neumann boundary traces $\left\{\left.\partial_{\nu} \varphi_{i j}\right|_{\Gamma_{1}}\right\}_{j=1}^{\ell_{i}}$ (respectively the $\ell_{i}$ Dirichlet boundary traces $\left.\left\{\left.\varphi_{i j}\right|_{\Gamma_{1}}\right\}_{j=1}^{\ell_{i}}\right)$ of the associated $\left(L_{2}(\Omega)\right.$-linearly independent) normalized eigenfunctions $\left\{\varphi_{i j}\right\}_{j=1}^{\ell_{i}}$ linearly independent also in $L_{2}\left(\Gamma_{1}\right)$, where $\Gamma_{1}$ is an arbitrary, open, connected portion of the boundary $\Gamma=\partial \Omega$ of positive measure?

This issue plays a critical role in, among other things, the solution of the purely boundary feedback stabilization problem (in particular, stability enhancement) of unstable parabolic problems, by means of a finite-dimensional boundary feedback, defined in terms of boundary traces in the feedback loop [L-T.2].

This problem was studied in [L-T.2] and represents the most demanding boundary feedback stabilization problem for parabolic equations defined on a bounded domain, where only (a portion of) the boundary is accessible to outside manipulations (sensors/controls). Its solution also rests on a positive answer to the aforementioned question. This will be further elaborated upon in Section I.2 below. The aim of Part I is to show that the answer to the question raised at the outset is in the affirmative. As we shall see, the solution proposed here hinges critically on a uniqueness property of over-determined elliptic problems. In the present section, we shall treat specifically the aforementioned question of linear independence of boundary traces of the (true) eigenfunctions in a more general setting, which involves a general second-order elliptic operator.

Let $\Omega$ be a bounded open domain in $\mathbb{R}^{n}$ with boundary $\Gamma=\partial \Omega$ assumed to be an $(n-1)$-dimensional manifold with $\Omega$ locally on one side of $\Gamma$. Let $\Gamma_{1}$ be an open, connected subset of $\Gamma$ of positive surface measure. Let $A(x, \partial)$ be a uniformly strongly elliptic operator of order two in $\Omega$, canonically $A=-\Delta$, of the form

$$
A(x, \partial) \equiv \sum_{|\alpha| \leq 2} a_{\alpha}(x) \partial^{\alpha}, \quad x \in \Omega,
$$

$x=\left[x_{1}, \ldots, x_{n}\right]$, with smooth real coefficients $a_{\alpha}(\cdot)$, where the symbol $\partial$ denotes differentiation.

REMARK 1.1. To ensure the validity of the critical unique continuation theorem (Carleman-Aronszajn-Cordes uniqueness theorem) to be invoked 
in the proof of Theorem 1D (or 1N) below, we may just assume that (i) the coefficients $a_{\alpha}$ are real-valued Lipschitz continuous functions for $|\alpha|=2$, and (ii) $a_{\alpha}$ are bounded for $|\alpha|<2$ [H, Vol. III, p. 3], [M, p. 60].

To the differential expression $A(x, \partial)$ in (1.1), we now associate suitable boundary conditions. We distinguish two basic cases.

Dirichlet B.C. case. Let $A_{D}$ denote the (closed) realization of $-A(x, \partial)$ (canonically, $-A=\Delta$ ) on $L_{2}(\Omega)$ with Dirichlet boundary conditions:

$$
\begin{aligned}
A_{D} f & =-A(x, \partial) f, \\
\mathcal{D}\left(A_{D}\right) & =H^{2}(\Omega) \cap H_{0}^{1}(\Omega)=\left\{f \in H^{2}(\Omega):\left.f\right|_{\Gamma}=0\right\} ; \\
A_{D} & : L_{2}(\Omega) \supset \mathcal{D}\left(A_{D}\right) \rightarrow L_{2}(\Omega) .
\end{aligned}
$$

Since $\Omega$ is bounded in $\mathbb{R}^{n}$, the closed operator $A_{D}$ has compact resolvent on $L_{2}(\Omega)$. Let

$$
\left\{\lambda_{i}^{D}, \varphi_{i j}^{D}\right\}, \quad i=1,2, \ldots ; j=1, \ldots, \ell_{i}^{D} ; \quad A_{D} \varphi_{i j}^{D}=\lambda_{i}^{D} \varphi_{i j}^{D},
$$

be the eigenvalues and corresponding (normalized) $L_{2}(\Omega)$-linearly independent eigenfunctions of $A_{D}$. Thus, in this section, $\ell_{i}^{D}$ denotes the geometric multiplicity of the eigenvalue $\lambda_{i}^{D}$.

Theorem 1D. Consider a fixed eigenvalue $\lambda_{i}^{D}$ (i.e., let $i$ be arbitrary but fixed) of the operator $A_{D}$, along with the corresponding set of $\ell_{i}^{D}$ linearly independent (normalized) eigenfunctions $\left\{\varphi_{i j}^{D}\right\}_{j=1}^{\ell_{i}^{D}}$ in $L_{2}(\Omega)$. Let $\Gamma_{1}$ be an open connected portion of the boundary $\Gamma$ of positive measure, which is assumed to be, say, of class $C^{2}$. Then

$$
\text { the system }\left\{\left.\partial_{\nu} \varphi_{i j}^{D}\right|_{\Gamma_{1}}\right\}_{j=1}^{\ell_{i}^{D}} \text { is linearly independent in } L_{2}\left(\Gamma_{1}\right) \text {. }
$$

Neumann case. Let $A_{N}$ be now the (closed) realization of $-A(x, \partial)$ (canonically, $\Delta$ ) on $L_{2}(\Omega)$, this time with Neumann boundary conditions:

$$
\begin{gathered}
A_{N} f \equiv-A(x, \partial) f, \quad \mathcal{D}\left(A_{N}\right)=\left\{f \in H^{2}(\Omega):\left.\frac{\partial f}{\partial \nu}\right|_{\Gamma}=0\right\} \\
A_{N}: L_{2}(\Omega) \supset \mathcal{D}\left(A_{N}\right) \rightarrow L_{2}(\Omega) .
\end{gathered}
$$

The closed operator $A_{N}$ has likewise compact resolvent on $L_{2}(\Omega)$. Let

$$
\left\{\lambda_{i}^{N}, \varphi_{i j}^{N}\right\}, \quad i=1,2, \ldots ; j=1, \ldots, \ell_{i}^{N} ; \quad A_{N} \varphi_{i j}^{N}=\lambda_{i}^{N} \varphi_{i j}^{N},
$$

be the eigenvalues and corresponding (normalized) $L_{2}(\Omega)$-linearly independent eigenfunctions of $A_{N}$ in $L_{2}(\Omega)$. Thus, here, $\ell_{i}^{N}$ denotes the geometric multiplicity of the eigenvalue $\lambda_{i}^{N}$.

TheOREM $1 \mathrm{~N}$. Consider the fixed eigenvalue $\lambda_{i}^{N}$ (i.e., let $i$ be arbitrary but fixed) of the operator $A_{N}$, along with the corresponding set of $\ell_{i}^{N}$ linearly independent (normalized) eigenfunctions $\left\{\varphi_{i j}^{N}\right\}_{j=1}^{\ell_{i}^{N}}$. Let $\Gamma_{1}$ be an open, connected portion of the boundary $\Gamma$ of positive measure which is assumed 
to be, say, of class $C^{2}$. Then

(1.7) the system $\left\{\left.\varphi_{i j}^{N}\right|_{\Gamma_{1}}\right\}_{j=1}^{\ell_{i}^{N}}$ is linearly independent in $L_{2}\left(\Gamma_{1}\right)$.

REMARK 1.2. Theorem $1 \mathrm{~N}$ admits a perfect counterpart with the Neumann boundary conditions associated to $A(x, \partial)$ replaced by the Robin boundary conditions.

Proof of Theorems $1 D$ and $1 N$. We write explicitly only the proof of Theorem 1D. The proof of Theorem $1 \mathrm{~N}$ is then the perfect counterpart, mutatis mutandis. We drop the superscript " $D$ " for simplicity of notation. To prove Theorem 1D, let as in [B-T], [B-L-T.1]

$$
\sum_{j=1}^{\ell_{i}} \alpha_{j} \partial_{\nu} \varphi_{i j}=\partial_{\nu}\left(\sum_{j=1}^{\ell_{i}} \alpha_{j} \varphi_{i j}\right)=\partial_{\nu} \varphi \equiv 0 \quad \text { on } \Gamma_{1},
$$

where we have defined the function $\varphi$ by

$$
\varphi \equiv \sum_{j=1}^{\ell_{i}} \alpha_{j} \varphi_{i j} \in L_{2}(\Omega)
$$

We must show that

$$
\alpha_{j} \equiv 0, \quad j=1, \ldots, \ell_{i} .
$$

To this end, we note that, by (1.3) and (1.9), $\varphi$ is an eigenfunction of $A_{D}$ with the same eigenvalue $\lambda_{i}: A_{D} \varphi=\lambda_{i} \varphi$. Thus, $\varphi$ satisfies homogeneous Dirichlet boundary conditions on all of $\Gamma:\left.\varphi\right|_{\Gamma}=0$, since so do the $\varphi_{i j}$ 's. Moreover, $\left.\partial_{\nu} \varphi\right|_{\Gamma_{1}}=0$ by (1.8). Thus, $\varphi$ satisfies the following over-determined elliptic eigenproblem:

$$
\left\{\begin{array}{l}
A(x, \partial) \varphi=\lambda_{i} \varphi \quad \text { in } \Omega \\
\left.\varphi\right|_{\Gamma} \equiv 0 ;\left.\quad \partial_{\nu} \varphi\right|_{\Gamma_{1}} \equiv 0
\end{array}\right.
$$

It is then well known that for a boundary $\Gamma$ of class, say, $C^{2}$, and coefficients as in Remark 1.1, as assumed, the over-determined elliptic eigenvalue problem $(1.11 \mathrm{a}-\mathrm{b})$ only has the zero solution:

$$
\varphi \equiv \sum_{j=1}^{\ell_{i}} \alpha_{j} \varphi_{i j} \equiv 0 \in L_{2}(\Omega) ; \quad \text { hence } \quad \alpha_{j}=0, \quad j=1, \ldots, \ell_{i} .
$$

The last implication on the coefficients $\alpha_{j}$ follows, since the $\varphi_{i j}$ 's are linearly independent in $L_{2}(\Omega)$. Thus (1.10) is established. The desired uniqueness property $\varphi \equiv 0$ in $L_{2}(\Omega)$ implied in (1.12) by the over-determined elliptic eigenvalue problem $(1.11 \mathrm{a}-\mathrm{b})$ is a corollary of well-known results (see e.g., a detailed discussion in [M, Chapter III, Sect. 19, pp. 59-61]: either the Aronszajn-Cordes uniqueness theorem [H, Vol. III, p. 3] or Carleman's theorem [C], [B-J-S, p. 162; p. 262], [M, pp. 59-61]), under regularity of the coef- 
ficients as in Remark 1.2, states that a solution $u \in H^{2}(\Omega)$ of a second-order elliptic problem which vanishes (to infinite order at some point, in particular) identically in an open interior subset $\omega$ of $\Omega: u \equiv 0$ in $\omega$, must vanish identically in $\Omega$. From this basic result, one then deduces the desired conclusion $\varphi \equiv 0$ for problem $(1.11 \mathrm{a}-\mathrm{b})$ (with boundary $\Gamma$ of class, say, $\left.C^{2}\right)$ in a classical way: by extending $\varphi$ by zero across $\Gamma_{1}$ outside $\Omega$ into a set $\omega_{\text {ext }}$, showing by integration by parts using the zero Cauchy data in (1.11b) that the extended function defined by $\varphi=$ solution of $(1.11 \mathrm{a}-\mathrm{b})$ in $\Omega$, and $\varphi \equiv 0$ in $\omega_{\text {ext }}$ satisfies $\varphi \in H^{2}(G)$, where $G=\Omega \cup \omega_{\text {ext }}$ [Kom, p. 75]. Then, the aforementioned Carleman's theorem applies on $G$ and yields $\varphi \equiv 0$ in $\Omega$, as desired. The proof is complete. [Another approach to obtaining uniqueness of the over-determined elliptic problem with zero Cauchy data on a portion of the boundary from the uniqueness theorem for solutions vanishing to infinite order at one point is given in $[\mathrm{S}-\mathrm{W}]$, under some smoothness of the coefficients: one flattens the boundary locally and performs an odd reflection across the boundary.]

REMARK 1.3. The proof of Theorem $1 \mathrm{~N}$ yields instead the following over-determined eigenvalue problem:

$$
\left\{\begin{array}{l}
A(x, \partial) \varphi=\lambda_{i} \varphi \quad \text { in } \Omega ; \\
\left.\varphi\right|_{\Gamma_{1}} \equiv 0 ;\left.\quad \partial_{\nu} \varphi\right|_{\Gamma} \equiv 0
\end{array}\right.
$$

again with zero Cauchy data on the portion $\Gamma_{1}$, which again allows for an extension by zero across $\Gamma_{1}$.

I.2. Implications of linear independence of boundary traces of eigenfunctions to the problem of boundary feedback stabilization of parabolic problems. In the present section, we point out the relevance of the results of the previous section on the linear independence of boundary traces of eigenfunctions. We keep the bounded open domain $\Omega$ in $\mathbb{R}^{n}$ as well as the elliptic operator $A(x, \partial)$ of Section I.1, Eqns. (1.1), (1.2). We assume, however, $\operatorname{dim} \Omega=n \geq 2$, as we shall need a full function space $L_{2}(\Gamma)$ to "counteract" the possibly large number of original unstable eigenvalues.

The unstable closed-loop parabolic system. As in [L-T.2], we consider the following closed-loop parabolic problem in the unknown $y(t, x)$ defined over $\Omega$ :

$$
\begin{cases}\frac{\partial y}{\partial t}(t, x)=-A(x, \partial) y(t, x) & \text { in } Q=(0, \infty] \times \Omega ; \\ y(0, x)=y_{0}(x) & \text { in } \Omega ; \\ \frac{\partial y}{\partial \nu}=\sum_{k=1}^{K}\left(\left.y(t, \cdot)\right|_{\Gamma}, w_{k}\right)_{\Gamma} g_{k} & \text { in } \Sigma=(0, \infty) \times \Gamma .\end{cases}
$$


Here, $w_{k}$ and $g_{k}$ are fixed boundary vectors in $L_{2}(\Gamma)$; the symbol $(\cdot, \cdot)_{\Gamma}$ denotes the inner product in $L_{2}(\Gamma)$. The boundary vectors $\left\{g_{k}\right\}_{k=1}^{K}$ are assumed to be linearly independent. Well-posedness of the closed-loop problem $(2.1 \mathrm{a}-\mathrm{c})$ is well known (even in the case where $\Gamma$ may have finitely many conical points [Kon].

Theorem 2.1 ([L-T.4]). The closed-loop feedback problem $(2.1 \mathrm{a}-\mathrm{c})$ is well posed in the following sense: its closed-loop feedback solutions $y\left(t ; y_{0}\right)$ can be expressed as $y\left(t ; y_{0}\right)=S_{F}(t) y_{0}, y_{0} \in L_{2}(\Omega), t \geq 0$, where $S_{F}(t)$ defines a (feedback) strongly continuous semigroup which is analytic and compact on $L_{2}(\Omega)$ for $t>0$, and whose generator has compact resolvent on $L_{2}(\Omega)$.

REMARK 2.1. The proof of [L-T.4] actually shows that the feedback semigroup $S_{F}(t)$ has the same properties listed in the statement of the theorem on all spaces $H^{3 / 2-\varepsilon}(\Omega), 0<\varepsilon \leq 3 / 2$. The boundary stabilization results of [L-T.1]-[L-T.3] are topologically consistent with the described regularity of feedback solutions. We also refer to [T.2] for regularity results, obtained by different techniques, that complement, but neither fully imply, nor are fully implied by, the above Theorem 2.1 [T.2, Remark 2.3]. Previously, stabilization problems for parabolic equations were given in [T.1], [T.2].

The most significant version of the problem at hand is when the free open loop system $\left(g_{k} \equiv 0\right)$ is unstable, in the sense that there are $I$ unstable distinct eigenvalues $\lambda_{1}, \ldots, \lambda_{I}$ of the $L_{2}(\Omega)$-realization $A_{N}$ of $(1.5 \mathrm{a}-\mathrm{b})$ associated with $-A(x, \partial)$ :

$$
\cdots \leq \operatorname{Re} \lambda_{I+2} \leq \operatorname{Re} \lambda_{I+1}<0 \leq \operatorname{Re} \lambda_{I} \leq \cdots \leq \operatorname{Re} \lambda_{2} \leq \operatorname{Re} \lambda_{1},
$$

where the eigenvalues of $A_{N}$ are numbered in order of decreasing real parts. The operator $A_{N}$ is the generator of a s.c., analytic semigroup on $L_{2}(\Omega)$.

Boundary feedback stabilization problem. This may be stated as follows: Find, if possible,

(i) appropriate boundary vectors $g_{k} \in L_{2}(\Gamma)$, as well as their minimum number, and

(ii) the weakest possible conditions on the vectors $w_{k} \in L_{2}(\Gamma), k=$ $1, \ldots, K$, to guarantee that the feedback semigroup $S_{F}(t)$ of Theorem 2.1 satisfies

$$
\left\|S_{F}(t) y_{0}\right\|_{L_{2}(\Omega)} \leq M e^{-\delta t}\left\|y_{0}\right\|_{L_{2}(\Omega)}, \quad \forall y_{0} \in L_{2}(\Omega),
$$

for some $M \geq 1$ and $\delta>0$ (perhaps, preassigned), or more generally,

$$
\left\|S_{F}(t) y_{0}\right\|_{H^{2 \sigma}(\Omega)} \leq M e^{-\delta t}\left\|y_{0}\right\|_{H^{2 \sigma}(\Omega)}, \quad \forall y_{0} \in H^{2 \sigma}(\Omega),
$$

for all $0 \leq \sigma<3 / 4$ (and $M \geq 1, \delta>0$, depending on $\sigma$ ). 
A solution of this problem is provided by [L-T.2]; see also [L-T.1], [L-T.3]. One of the assumptions required - given below - represents the link with the problem of linear independence of boundary traces of the preceding section. We shall assume the following further hypothesis, which is typically satisfied in classical parabolic equations where $A_{D}$ or $A_{N}$ are self-adjoint or normal operators:

$$
\text { The distinct unstable eigenvalues }\left\{\lambda_{i}\right\}_{i=1}^{I} \text { are semisimple; }
$$

that is, the restriction of the free dynamics generator $A_{N}$ in $(1.5 \mathrm{a}-\mathrm{b})$ is diagonalizable (or semisimple [Ka, p. 43]) when restricted to the finitedimensional unstable subspace $Y_{u}$ of $L_{2}(\Omega)$ generated by the eigenvectors of the unstable eigenvalues $\left\{\lambda_{i}\right\}_{i=1}^{I}$ in (2.2). This means that for each unstable eigenvalue $\lambda_{i}, i=1, \ldots, I$, the algebraic and geometric multiplicities $\ell_{i}$ coincide. Thus, each such eigenvalue $\lambda_{i}$ has $\ell_{i}$ linearly independent (normalized) eigenfunctions $\left\{\varphi_{i j}\right\}_{j=1}^{\ell_{i}}$ in $L_{2}(\Omega)$. Moreover, the unstable subspace $Y_{u}$ is $\operatorname{span}\left\{\varphi_{i j}: i=1, \ldots, I ; j=1, \ldots, \ell_{i}\right\}$. Thus, let

$$
A_{N} \varphi_{i j}=\lambda_{i} \varphi_{i j} ; \quad A_{N}^{*} \varphi_{i j}^{*}=\bar{\lambda}_{i} \varphi_{i j}^{*} \quad i=1, \ldots, I ; j=1, \ldots, \ell_{i},
$$

$\ell_{i}=$ geometric and algebraic multiplicity of $\lambda_{i}$ or $\bar{\lambda}_{i}$, be the eigenvalues/ eigenvectors of $A_{N}$ and of its $L_{2}(\Omega)$-adjoint $A_{N}^{*}$. It is well known [Ka, p. 51] that $\left\{\varphi_{i j}\right\}$ and $\left\{\varphi_{i j}^{*}\right\}$ can be chosen to form bi-orthogonal sequences; that is,

$$
\left(\varphi_{i j}, \varphi_{h k}^{*}\right)_{L_{2}(\Omega)}= \begin{cases}1 & \text { if } i=h ; j=k \\ 0 & \text { otherwise }\end{cases}
$$

Thus, any vector $y \in Y_{u}$ admits the unique expansion

$$
Y_{u} \ni y=\sum_{i, j}\left(y, \varphi_{i j}^{*}\right)_{L_{2}(\Omega)} \varphi_{i j}, \quad i=1, \ldots, I ; j=1, \ldots, \ell_{i} .
$$

In this case, a condition imposed in [L-T.2, p. 311] on the vectors $w_{k}$ to achieve uniform feedback stabilization of problem $(2.1 \mathrm{a}-\mathrm{c})$ is that the rank of the $K \times \ell_{i}$ matrix

$$
W_{i}=\left[\begin{array}{cccc}
\left(w_{1},\left.\varphi_{i 1}^{*}\right|_{\Gamma_{1}}\right)_{\Gamma_{1}} & \left(w_{1},\left.\varphi_{i 2}^{*}\right|_{\Gamma_{1}}\right)_{\Gamma_{1}} & \ldots & \left(w_{1},\left.\varphi_{i \ell_{i}}^{*}\right|_{\Gamma_{1}}\right)_{\Gamma_{1}} \\
\left(w_{2},\left.\varphi_{i 1}^{*}\right|_{\Gamma_{1}}\right)_{\Gamma_{1}} & \left(w_{2},\left.\varphi_{i 2}^{*}\right|_{\Gamma_{1}}\right)_{\Gamma_{1}} & \ldots & \left(w_{2},\left.\varphi_{i \ell_{i}}^{*}\right|_{\Gamma_{1}}\right)_{\Gamma_{1}} \\
\vdots & \vdots & & \vdots \\
\left(w_{K},\left.\varphi_{i 1}^{*}\right|_{\Gamma_{1}}\right)_{\Gamma_{1}} & \left(w_{K},\left.\varphi_{i 2}^{*}\right|_{\Gamma_{1}}\right)_{\Gamma_{1}} & \ldots & \left(w_{K},\left.\varphi_{i \ell_{i}}^{*}\right|_{\Gamma_{1}}\right)_{\Gamma_{1}}
\end{array}\right]
$$

be full, where $K \geq \ell_{i}$ has been preliminarily chosen, that is,

$$
\operatorname{rank} W_{i}=\ell_{i}, \quad i=1, \ldots, I .
$$


The algebraic condition (2.10) can only be fulfilled if, for each $i=$ $1, \ldots, I-1$, the Dirichlet traces $\left\{\left.\varphi_{i j}^{*}\right|_{\Gamma_{1}}\right\}_{j=1}^{\ell_{i}}$ of the eigenfunctions of $A_{N}^{*}$ are linearly independent. Theorem $1 \mathrm{~N}$ (as applied to $A_{N}^{*}$ ) guarantees that this is always possible, in which case the vectors $\left\{w_{1}, \ldots, w_{K}\right\}$ can be chosen in infinitely many ways.

\section{PART II: THE STOKES/OSEEN OPERATORS}

Part I has shown that the linear independence of the traces of the eigenfunctions critically hinges on a unique continuation property from the boundary of the corresponding boundary value problem. This result is well known for second-order elliptic operators, as noted in the proof of Theorem 1D. Apparently, no similar result is known for the Stokes operator (the author inquired about that among several senior experts on such operators worldwide in the Spring of 2008); see Remark 1.1 below. Accordingly, such uniqueness property is the focus of the present Part II. It is motivated by similar boundary stabilization (or stability enhancement) problems for linear/linearized Navier-Stokes equations [B-L-T.1]-[B-L-T.3], [L-T.6].

II.3. The unique continuation property from the boundary of the Stokes and Oseen problems. Let $\Omega$ be an open bounded domain in $\mathbb{R}^{d}$; we shall focus on the cases $d=2,3$ of physical relevance. We assume that $\Gamma=\partial \Omega$ is of class $C^{2}$. With reference to [Te, p. 39], let $\{\varphi, p\}$, with $\varphi \in$ $\left(H^{2}(\Omega)\right)^{d}, p \in H^{1}(\Omega)$, be a solution of the over-determined Stokes problem

$$
\begin{cases}(-\Delta) \varphi+\nabla p=\lambda \varphi & \text { in } \Omega, \lambda>0 \\ \operatorname{div} \varphi \equiv 0 & \text { in } \Omega \\ \left.\varphi\right|_{\Gamma}=0 ; & \\ \left.\frac{\partial \varphi}{\partial \nu}\right|_{\Gamma}=0, & \end{cases}
$$

where we have normalized the viscosity coefficient $\nu_{0}=1$.

TheOREM 3.1. The over-determined problem (3.1a-d) implies

$$
p \equiv \text { const } \quad \text { in } \Omega ; \quad \varphi \equiv 0 \quad \text { in } \Omega .
$$

The author has several different proofs of this result [T.4], some of which work also for the corresponding Oseen problem, provided that the related equilibrium (steady-state) solution $y_{e}$ is sufficiently small, say, in the $\left(W^{1, \infty}(\Omega)\right)^{d}$-norm. The Oseen equation is given by

$$
(-\Delta) \varphi+L_{e}(\varphi)+\nabla p=\lambda \varphi \quad \text { in } \Omega, \quad \varphi \in\left(H^{2}(\Omega)\right)^{d}, p \in H^{1}(\Omega),
$$


where the first-order differential operator $L_{e}(\varphi)$ is defined by

$$
L_{e}(\varphi)=\left(y_{e} \cdot \nabla\right) \varphi+(\varphi \cdot \nabla) y_{e},
$$

where the equilibrium solution $y_{e}$ is part of the pair $\left\{y_{e}, p_{e}\right\} \in\left(\left(H^{2}(\Omega)\right)^{d} \cap V\right)$ $\times H^{1}(\Omega)$ that satisfies the problem

$$
\begin{cases}(-\Delta) y_{e}+\left(y_{e} \cdot \nabla\right) y_{e}+\nabla p_{e}=f_{e} & \text { in } \Omega ; \\ \operatorname{div} y_{e} \equiv 0 & \text { in } \Omega ; \\ y_{e} \equiv 0 & \text { in } \Gamma,\end{cases}
$$

with $f_{e}$ an external force. The equilibrium solution is known to exist (with the given regularity) for $d=2,3$ [C-F, Thm. 7.3, p. 18]. Moreover, $V$ is the space [C-F, p. 19], [Te, p. 18]:

$$
V \equiv\left\{y \in\left(H_{0}^{1}(\Omega)\right)^{d}: \operatorname{div} y \equiv 0 \text { in } \Omega\right\}
$$

with norm

$$
\|y\|_{V}=\left\{\int_{\Omega}|\nabla y(x)|^{2} d \Omega\right\}^{1 / 2}
$$

The "elementary" proof given in Section II.5 works explicitly for the Stokes problem. In Section II.6, we shall give the following topological result (Theorem 6.3) for the Oseen problem (consisting of (3.3), (3.4), (3.1b-d)): the set of equilibrium solutions $y_{e}$ for which the unique continuation property (that is, the conclusion (3.2) of Theorem 3.1) holds true is open in the space $\left(W^{1, \infty}(\Omega)\right)^{d}$.

A more standard unique continuation property — valid also for the Oseen problem - is as follows.

Theorem 3.2. Let $\varphi \in\left(H^{2}(\Omega)\right)^{d}, p \in H^{1}(\Omega)$ be a solution of the overdetermined Oseen problem

$$
\begin{cases}(-\Delta) \varphi+L_{e}(\varphi)+\nabla p=\lambda \varphi & \text { in } \Omega ; \\ \operatorname{div} \varphi=0 & \text { in } \Omega ; \\ \left.\varphi\right|_{\Gamma_{1}}=0, \quad\left[\frac{\partial \varphi}{\partial \nu}-p \nu\right]_{\Gamma_{1}}=0, & \end{cases}
$$

where $\Gamma_{1}$ is an open subset of $\Gamma$ of positive surface measure, and $\nu$ is the unit outward normal. Then

$$
\varphi \equiv 0 \quad \text { and } \quad p \equiv \text { const in } \Omega .
$$

The proof will be sketched in Section II.7 below, and is based on [B-L-T.1, Sect. 3.6].

In Section II.4, we shall consider an important implication of the unique continuation property of Theorems 3.1 and 3.2, respectively. 
Implications on the boundary feedback stabilization problem for the (dynamic) linearized and nonlinear Navier-Stokes equations [B-L-T.1][B-L-T.3], [R]. In the present Part II (dealing with static linear/linearized fluid equations derived from the Navier-Stokes equations), unique continuation results for corresponding over-determined problems from the boundary are given in the following cases:

(1) The Stokes over-determined problem $(3.1 \mathrm{a}-\mathrm{d})$, as expressed by Theorem 3.1 for $d=2,3$ (a proof of which is given in Section II.5). For the case $d=2$, an alternative proof is given in Section II.8 (see Proposition 8.2).

(2) The Oseen over-determined problem $(6.27 \mathrm{a}-\mathrm{c})$ below (that is, (3.4), $(3.1 \mathrm{~b}-\mathrm{d}))$, but this time only for equilibrium solutions $y_{e}$ in a sufficiently small $\left(W^{1, \infty}(\Omega)\right)^{d}$-neighborhood of the origin $y_{e} \equiv 0$ (Stokes problem); or, potentially more general, in a sufficiently small $\left(W^{1, \infty}(\Omega)\right)^{d}$-neighborhood of a "good" equilibrium solution $y_{e}$, where the unique continuation problem holds true. This is expressed by Theorem 6.3 .

(3) The Oseen over-determined problem, now in the form $(3.7 \mathrm{a}-\mathrm{c})$ [involving also $p$ on the boundary] in full generality (Theorem 3.2).

In addition, a unique continuation result for the Oseen problem $(6.27 \mathrm{a}-\mathrm{c})$, in full generality regarding the equilibrium solution $y_{e}$, but complemented by the additional condition that $\varphi \cdot \tau \equiv 0$ in an (arbitrarily small) collar $\omega$ of the boundary $\Gamma$, where $\tau$ is a vector "tangential" at any point in $\omega$, is given in [L-T.6]. Also, additional proofs (by Rellich multiplier; by Carleman estimates) of the Oseen over-determined problem $(6.27 \mathrm{a}-\mathrm{c})$ for sufficiently small equilibrium solutions $y_{e}$ (in the $\left(W^{1, \infty}(\Omega)\right)^{d}$-norm) are given in [T.4].

All these results have critical implications in the treatment of the boundary feedback stabilization problem for the dynamic Oseen equations (linearized Navier-Stokes equations), as well as for the (fully nonlinear) NavierStokes equation itself, as treated in [B-L-T.1]-[B-L-T.3], [R]. More precisely:

(a) The results of [B-L-T.1]-[B-L-T.3] hold true with tangential boundary feedback in the case of the Stokes or Oseen dynamic equations, with $y_{e}$ sufficiently small in the latter case, thus providing results on enhancement of stability properties by tangential boundary control. To this end, Theorems 3.1 and 6.3 are needed.

(b) The tangential boundary control treatment of [B-L-T.1, Section 3.6], when integrated with that of $[R]$, in the presence also of a normal component, now yields - by relying on the unique continuation Theorem 3.2, hence on Theorem 4.2 below-corresponding boundary feedback stabilization results for general $y_{e}$, with boundary feedback control not necessarily tangential.

This is further elaborated and enhanced in Section II.4. 
REMARK 3.1. In the case of tangential boundary stabilization of NavierStokes equations treated in [B-L-T.1] (as well as in [B-L-T.2]-[B-L-T.3]), the argument to establish the unique continuation property given in [B-L-T.1, Section 3.6, p. 55] is not conclusive in writing [B-L-T.1, Eqn. (3.6.24a), p. 55], unless the pressure is constant on the portion $\Gamma_{1}$ of the boundary, where the Cauchy data vanish. (We thank J. P. Raymond for pointing out this gap to the authors of [B-L-T.1] in March 2008.) However, the desired conclusion of that argument: $\tilde{\varphi}^{*} \equiv 0, \tilde{p}^{*} \equiv 0$ in $\tilde{\Omega}=\Omega \cup \omega$-hence the critical linear independence of the boundary traces $\left\{\left.\partial_{\nu} \varphi_{i j}^{*}\right|_{\Gamma}\right\}_{j=1}^{\ell_{i}}$ [B-L-T.1, Eqn. (3.6.20a), p. 54] is indeed true at least in the aforementioned cases (1), (2), which are relevant to the stabilization problem for the (Oseen, hence) Navier-Stokes equations by a tangential boundary feedback control. Thus, Theorem 4.1 of Section II.4 (in the Stokes case), as well as Theorem 6.3/Corollary 6.4 of Section II.6 (in the case of the Oseen equation with small equilibrium solution $y_{e}$ in the $\left(W^{1, \infty}(\Omega)\right)^{d}$-norm) do establish in these two cases (1) and (2) the required linear independence of the boundary traces $\left\{\left.\partial_{\nu} \varphi_{i j}^{*}\right|_{\Gamma}\right\}_{j=1}^{\ell_{i}}$ in [B-L-T.1, Eqn. (3.6.20a), p. 54].

\section{II.4. Implication: Linear independence of boundary traces of eigenfunctions of the Stokes or Oseen problems}

Stokes problem. Again, with reference to [Te, p. 39], it is well known that the Stokes problem over a bounded domain $\Omega$ is self-adjoint, with compact resolvent, and hence possesses an orthonormal sequence of eigenfunctions $\left\{w_{j}\right\}_{j=1}^{\infty}$ corresponding to the positive eigenvalues $\lambda_{j}>0, \lambda_{j} \rightarrow$ $+\infty$ as $j \rightarrow+\infty$, counted with multiplicity. Thus, for $C^{2}$-boundary $\Gamma$, we have

$$
w_{j} \in\left(H^{2}(\Omega)\right)^{d}, \quad p_{j} \in H^{1}(\Omega), \quad j=1,2, \ldots,
$$

and

$$
\begin{cases}(-\Delta) w_{j}+\nabla p_{j}=\lambda_{j} w_{j} & \text { in } \Omega \\ \operatorname{div} w_{j} \equiv 0 & \text { in } \Omega ; \\ \left.w_{j}\right|_{\Gamma}=0 . & \end{cases}
$$

[In general, with boundary $\Gamma$ of class $C^{m}$ for an integer $m \geq 2$, we have $w_{j} \in\left(H^{m}(\Omega)\right)^{d}, p_{j} \in H^{m-1}(\Omega)$; while for boundary $\Gamma$ of class $C^{\infty}$, we have $\left.w_{j} \in\left(C^{\infty}(\bar{\Omega})\right)^{d}, p_{j} \in C^{\infty}(\bar{\Omega}), j=1,2, \ldots[\mathrm{Te}, \mathrm{p} .39].\right]$

Henceforth, we denote by $\lambda_{i}$ the distinct eigenvalues and by $\left\{w_{i j}\right\}_{j=1}^{\ell_{i}}$ the corresponding normalized $\left.L_{2}(\Omega)\right)^{d}$-linearly independent eigenfunctions, where $\ell_{i}$ is the geometric (= algebraic) multiplicity of the eigenvalue $\lambda_{i}$. As a consequence of Theorem 3.1, we obtain 
THEOREM 4.1. With reference to the aforementioned eigenproblem $(4.2 \mathrm{a}-\mathrm{c})$ and the above notation,

$$
\text { the system }\left\{\left.\partial_{\nu} w_{i j}\right|_{\Gamma}\right\}_{j=1}^{\ell_{i}} \text { is linearly independent in }\left(L_{2}(\Gamma)\right)^{d} \text {. }
$$

Proof. Let (as in the proof of Theorem 1D, Part I)

$$
\sum_{j=1}^{\ell_{i}} \alpha_{j} \partial_{\nu} w_{i j}=\partial_{\nu}\left(\sum_{j=1}^{\ell_{i}} \alpha_{j} w_{i j}\right)=\partial_{\nu} w \equiv 0 \quad \text { on } \Gamma,
$$

where we have defined

$$
w=\sum_{j=1}^{\ell_{i}} \alpha_{j} w_{i j} \in\left(H^{2}(\Omega)\right)^{d} .
$$

We must show that

$$
\alpha_{j} \equiv 0, \quad j=1, \ldots, \ell_{i} .
$$

To this end, we note that by (4.2b), (4.2c) used in (4.5), we have $\operatorname{div} w \equiv 0$ in $\Omega$, and $\left.w\right|_{\Gamma}=0$. Thus, $w$ satisfies problem (3.1a-d) with $\varphi=w$ and $p=\sum_{j=1}^{\ell_{i}} p_{j}$. Application of the uniqueness Theorem 3.1 then yields

$$
w \equiv \sum_{j=1}^{\ell_{i}} \alpha_{j} w_{i j}=0 \quad \text { in }\left(L_{2}(\Omega)\right)^{d}, \quad \text { hence } \quad \alpha_{j} \equiv 0, \quad j=1, \ldots, \ell_{i},
$$

since the $\left\{w_{i j}\right\}_{j=1}^{\ell_{i}}$ are linearly independent in $\left(L_{2}(\Omega)\right)^{d}$. Theorem 4.1 is proved.

The Oseen problem. We consider the corresponding eigenvalue problem for the Oseen problem on $\Omega$ with $C^{2}$-boundary $\Gamma$. Let $\lambda_{i} \in \mathbb{C}$ be the distinct eigenvalues and $\left\{w_{i j}\right\}_{j=1}^{\ell_{i}}$ be the corresponding normalized $\left(L_{2}(\Omega)\right)^{d_{-}}$ linearly independent eigenfunctions, where $\ell_{i}$ denotes the geometric multiplicity. Thus, for $w_{i j} \in\left(H^{2}(\Omega)\right)^{d}$ and $p_{i j} \in H^{1}(\Omega)$, as in (4.1) we have

$$
\begin{cases}(-\Delta) w_{i j}+L_{e}\left(w_{i j}\right)+\nabla p_{i j}=\lambda_{j} w_{i j} & \text { in } \Omega ; \\ \operatorname{div} w_{i j}=0 & \text { in } \Omega ; \\ \left.w_{i j}\right|_{\Gamma}=0 . & \end{cases}
$$

TheOREM 4.2. With reference to $(4.8 \mathrm{a}-\mathrm{c})$,

$$
\begin{array}{r}
\text { the system }\left\{\left[\partial_{\nu} w_{i j}-p_{i j} \nu\right]_{\Gamma_{1}}\right\}_{j=1}^{\ell_{1}} \\
\text { is linearly independent in }\left(L_{2}\left(\Gamma_{1}\right)\right)^{d},
\end{array}
$$

where $\Gamma_{1}$ is an open subset of $\Gamma$ of positive surface measure.

The proof of Theorem 4.2 is the same as that of Theorem 4.1, except that it invokes the unique continuation Theorem 3.2, rather than Theorem 3.1. 
REMARK 4.1. We make reference to [B-L-T.1, Section 3.6]. If one replaces the matrices $W_{i}$ in [B-L-T.1, (3.6.10), p. 52] with the matrices

$$
\tilde{W}_{i}=\left[\begin{array}{ccc}
\left(w_{1}, \partial_{\nu} \varphi_{i 1}^{*}-p_{i 1} \nu\right)_{\Gamma} & \cdots & \left(w_{k}, \partial_{\nu} \varphi_{i 1}^{*}-p_{i 1} \nu\right)_{\Gamma} \\
\vdots & & \vdots \\
\left(w_{1}, \partial_{\nu} \varphi_{i \ell_{i}}^{*}-p_{i \ell_{i}} \nu\right)_{\Gamma} & \cdots & \left(w_{k}, \partial_{\nu} \varphi_{i 1}^{*}-p_{i 1} \nu\right)_{\Gamma}
\end{array}\right]
$$

of size $\ell_{i} \times k, i=1, \ldots, M$, then the required condition

$$
\operatorname{rank} \tilde{W}_{i}=\ell_{i}, \quad i=1, \ldots, M,
$$

can be satisfied with infinitely many choices of the vector $\left\{w_{1}, \ldots, w_{K}\right\}$, $K \geq N$, precisely by invoking Theorem 4.2, which is critically based on Theorem 3.2. This case corresponds by combining [B-L-T.1] with [R] to the boundary stabilization problem for the Navier-Stokes equations, with not necessarily tangential feedback control.

\section{II.5. Proof of Theorem 1.1}

SteP 0: Preliminaries ([L-T.1, Appendix 3C, p. 297]). The following considerations are actually local in character, and we may as well focus on a portion $\tilde{\Gamma}$ of $\Gamma$. Let $\eta \in \tilde{\Gamma}$, of class $C^{2}$. Let $\nu(\eta)$ denote the unit outward normal vector at $\eta$. On the tangent plane $M_{\eta}$ of $\Gamma$ at $\eta$, we let $\left[\tau_{1}(\eta), \tau_{2}(\eta)\right]$ denote an orthonormal system of tangent vectors
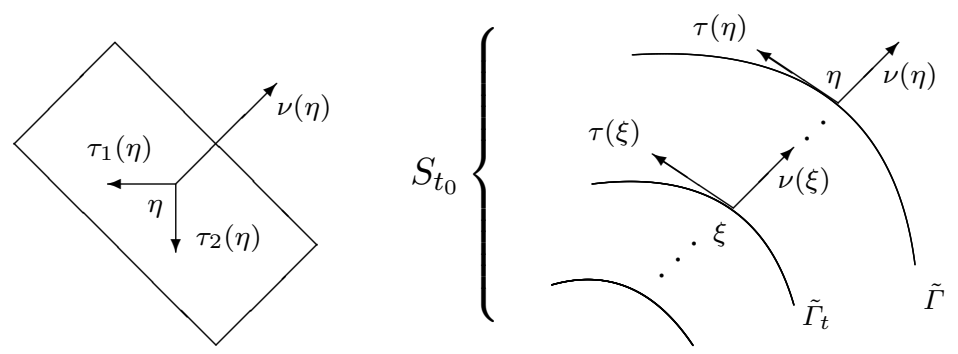

We then define the vector or point in $\Omega$ :

$$
\xi=r(t ; \eta)=\eta+t \nu(\eta), \quad-t_{0}<t<0, \eta \in \tilde{\Gamma},
$$

$\left|t_{0}\right|$ sufficiently small, which for $t$ fixed and $\eta$ running over $\tilde{\Gamma}$, describes the parallel translation surface $\tilde{\Gamma}_{t}$ of $\tilde{\Gamma}$ in $\Omega$; moreover, as $t$ runs over $\left(-t_{0}, 0\right)$, the family of surfaces $\tilde{\Gamma}_{t}$ sweeps a collar, or strip, $S_{t_{0}}$ of $\tilde{\Gamma}$ :

$$
\tilde{\Gamma}_{t}=\{r(t ; \eta): \eta \in \tilde{\Gamma}\}, \quad S_{t_{0}}=\bigcup_{-t_{0}<t<0} \tilde{\Gamma}_{t} .
$$


The map $\eta \in \tilde{\Gamma} \mapsto \xi \in S_{t_{0}}$ is one-to-one (the Jacobian is $\neq 0$ ). For each $\eta \in \tilde{\Gamma}$ and corresponding $\xi=\eta+t \nu(\eta) \in S_{t_{0}}$, we let $\nu(\xi)$ be the unit outward normal to the surface $\tilde{\Gamma}_{t}$ passing through $\xi$, and let $\left[\tau_{1}(\xi), \tau_{2}(\xi)\right]$ be the corresponding orthonormal system of tangent vectors. Thus, we have

$$
\begin{aligned}
\nu(\xi)=\nu(\eta), \quad\left[\tau_{1}(\xi), \tau_{2}(\xi)\right]=[ & \left.\tau_{1}(\eta), \tau_{2}(\eta)\right] \\
& \text { for } \xi=\eta+t \nu(\eta),-t_{0}<t<0,
\end{aligned}
$$

that is, the normal unit vector $\nu(\eta)$ at the boundary point $\eta \in \tilde{\Gamma}$ generates a constant vector field $\nu(\xi)$ for all points $\xi$ of the normal line to $\eta$ in the collar; and similarly for the orthonormal system $\left[\tau_{1}(\eta), \tau_{2}(\eta)\right]$ of tangent vectors. In this way, smooth vector fields $\nu(\xi)$ and $\left[\tau_{1}(\xi), \tau_{2}(\xi)\right]$ are defined at all points $\xi$ of the collar, by parallel translation of $\nu(\eta)$ and the pair $\left[\tau_{1}(\eta), \tau_{2}(\eta)\right], \eta \in \tilde{\Gamma}$, along the normal line to $\eta$. Thus, we may define the normal derivative and tangential derivatives of a sufficiently smooth vector $w=\left[w_{1}, \ldots, w_{d}\right], d=2,3$, to $\tilde{\Gamma}_{t}$ for each point $\xi=\eta+t \nu(\eta)$ of the collar $S_{t_{0}}$ besides the case $\eta \in \tilde{\Gamma}$ :

$$
\begin{gathered}
\frac{\partial w}{\partial \nu}(\xi)=\nabla w(\xi) \cdot \nu(\xi) ; \quad \frac{\partial w}{\partial \tau_{i}}(\xi)=\nabla w(\xi) \cdot \tau_{i}(\xi), \quad i=1,2, ; \\
\nabla_{\tau} w(\xi)=\frac{\partial w}{\partial \tau_{1}}(\xi) \cdot \tau_{1}(\xi)+\frac{\partial w}{\partial \tau_{2}}(\xi) \cdot \tau_{2}(\xi) ; \\
\nabla w(\xi)=\frac{\partial w}{\partial \nu}(\xi) \cdot \nu(\xi)+\nabla_{\tau} w(\xi) .
\end{gathered}
$$

STEP 1. Given a vector $w=\left[w_{1}, \ldots, w_{d}\right], d=2,3$, sufficiently smooth, the following two relations are well known to hold true pointwise at each point $\xi=Q$ of the collar of $\Gamma$ :

(a) [L-T.5, Prop. 3C.6, p. 305], [S-Z, Proposition 2.68, p. 94]:

$$
\left.\Delta w\right|_{Q}=\left.\frac{\partial^{2} w}{\partial \nu^{2}}\right|_{Q}+\left.\Delta_{\Gamma(\xi)} w\right|_{Q}+\left[\left(\frac{\partial w}{\partial \nu}\right)(\operatorname{div} \nu)\right]_{Q},
$$

where $\Delta_{\Gamma}$ is the tangential Laplacian $\left(\left.\Delta_{\Gamma} w\right|_{Q}=\left.\frac{\partial^{2} w}{\partial \tau^{2}}\right|_{Q}\right.$ when $d=2$, where $\tau=\left[-\nu_{2}, \nu_{1}\right]$ is the corresponding unit tangential vector).

(b) [A-T, Prop. A.1, Appendix A] For $d=2,3$,

$$
\begin{aligned}
{[\operatorname{div} w]_{Q} } & =\left[\frac{\partial w_{1}}{\partial x_{1}}+\cdots+\frac{\partial w_{d}}{\partial x_{d}}\right]_{Q} \\
& =\left[\frac{\partial w}{\partial \nu} \cdot \nu\right]_{Q}+\left[\frac{\partial w}{\partial \tau_{1}} \cdot \tau_{1}\right]_{Q}+\left[\frac{\partial w}{\partial \tau_{2}} \cdot \tau_{2}\right]_{Q}
\end{aligned}
$$


(c) [L-T.5, Eqn. 3C.68, p. 309] The following property holds true:

$$
\frac{\partial}{\partial \tau_{i}} \frac{\partial f}{\partial \nu}=\frac{\partial}{\partial \nu} \frac{\partial f}{\partial \tau_{i}}+(\operatorname{div} \nu) \frac{\partial f}{\partial \tau_{i}} \quad \text { on } \Gamma, i=1,2 .
$$

Thus, the commutator of $\partial / \partial \tau_{i}$ and $\partial / \partial \nu$ is a first-order tangential operator.

STEP 2. From (5.6), we readily obtain

Lemma 5.1. Let $w \in C^{1}(\bar{\Omega})$ and let $\Gamma_{1}$ be any portion of $\Gamma$. Then the following implication holds true:

$$
\left.\begin{array}{rl}
\left.w\right|_{\Gamma_{1}} \equiv 0 \\
{[\operatorname{div} w]_{\Gamma_{1}} \equiv 0}
\end{array}\right\} \Rightarrow \begin{array}{r}
\left.\frac{\partial w}{\partial \nu}\right|_{\Gamma_{1}} \cdot \nu=0, \\
\quad \text { i.e., } \quad \nabla w \cdot \nu=\left.\frac{\partial w}{\partial \nu}\right|_{\Gamma_{1}} \text { is tangential to } \Gamma_{1} .
\end{array}
$$

Proof. Invoke (5.6) on $\Gamma_{1}$, where $\left.\frac{\partial w}{\partial \tau_{i}}\right|_{\Gamma_{1}}=0, i=1,2$, since $\left.w\right|_{\Gamma_{1}}=0$.

Refer also to [B-L-T.1, Lemma 3.3.1] for a related result.

LEMMA 5.2. In the notation of the preliminaries, we have

$$
\left.\left.\varphi\right|_{\Gamma} \equiv 0 \Rightarrow \operatorname{div}\left(\frac{\partial \varphi}{\partial \nu}\right)\right|_{\Gamma}=\left[\frac{\partial}{\partial \nu}(\operatorname{div} \varphi)\right]_{\Gamma}
$$

i.e., div and $\partial / \partial \nu$ commute on $\Gamma$.

Proof. We use (5.6) with $w=\partial \varphi / \partial \nu$. We get on $\Gamma$ :

$$
\begin{aligned}
{\left[\operatorname{div}\left(\frac{\partial \varphi}{\partial \nu}\right)\right]_{\Gamma}=} & \left.\frac{\partial^{2} \varphi}{\partial \nu^{2}}\right|_{\Gamma} \cdot \nu+\left.\frac{\partial}{\partial \tau_{1}} \frac{\partial \varphi}{\partial \nu}\right|_{\Gamma} \cdot \tau_{1}+\left.\frac{\partial}{\partial \tau_{2}} \frac{\partial \varphi}{\partial \nu}\right|_{\Gamma} \cdot \tau_{2} \\
\stackrel{(5.7)}{=} & \left.\frac{\partial^{2} \varphi}{\partial \nu^{2}}\right|_{\Gamma} \cdot \nu+\left[\frac{\partial}{\partial \nu} \frac{\partial \varphi}{\partial \tau_{1}}\right]_{\Gamma} \cdot \tau_{1}+\left[\frac{\partial}{\partial \nu} \frac{\partial \varphi}{\partial \tau_{2}}\right]_{\Gamma} \cdot \tau_{2} \\
& +\left[(\operatorname{div} \nu) \frac{\partial \varphi}{\partial \tau_{1}}\right]_{\Gamma} \cdot \tau_{1}+\left[(\operatorname{div} \nu) \frac{\partial \varphi}{\partial \tau_{2}}\right]_{\Gamma} \cdot \tau_{2} \\
= & \left\{\frac{\partial}{\partial \nu}\left[\frac{\partial \varphi}{\partial \nu} \cdot \nu+\frac{\partial \varphi}{\partial \tau_{1}} \cdot \tau_{1}+\frac{\partial \varphi}{\partial \tau_{2}} \cdot \tau_{2}\right]\right\}_{\Gamma} \\
& +\left.(\operatorname{div} \nu) \frac{\partial \varphi}{\partial \tau_{1}}\right|_{\Gamma} \cdot \tau_{1}+\left.(\operatorname{div} \nu) \frac{\partial \varphi}{\partial \tau_{2}}\right|_{\Gamma} \cdot \tau_{2} \\
\stackrel{(5.6)}{=} & \left.\frac{\partial}{\partial \nu}(\operatorname{div} \varphi)\right|_{\Gamma}
\end{aligned}
$$

where $\left.\frac{\partial \varphi}{\partial \tau_{i}}\right|_{\Gamma}=0$ since $\left.\varphi\right|_{\Gamma}=0$ by assumption. 
Corollary 5.3. Via Lemma 5.2, in the same notation,

$$
\left.\begin{array}{rl}
\left.\varphi\right|_{\Gamma} & \equiv 0 \\
\operatorname{div} \varphi & \equiv 0 \text { in a collar of } \Gamma
\end{array}\right\} \Rightarrow\left[\operatorname{div}\left(\frac{\partial \varphi}{\partial \nu}\right)\right]_{\Gamma}=\left[\frac{\partial}{\partial \nu}(\operatorname{div} \varphi)\right]_{\Gamma}=0 .
$$

StEP 3. Continuing with the proof of Theorem 3.1, we have

Proposition 5.4. The Stokes problem (3.1a-c) of Part II [with no use of (3.1d)] implies the following problem for the pressure $p$ :

$$
\begin{cases}\Delta p=0 & \text { in } \Omega \\ \left.\frac{\partial p}{\partial \nu}\right|_{\Gamma}=\Delta \varphi \cdot \nu=\frac{\partial^{2} \varphi}{\partial \nu^{2}} \cdot \nu & \text { on } \Gamma .\end{cases}
$$

Proof. In fact, (5.11a) is obtained as usual by taking "div" across (3.1a) and using (3.1b). Moreover, (5.11b), in the preliminary form $\left.\frac{\partial p}{\partial \nu}\right|_{\Gamma}=\Delta \varphi \cdot \nu$ on $\Gamma$, is obtained by restricting (3.1a) to $\Gamma$, taking the dot product with $\nu$ and using (3.1c). Moreover, we obtain $\Delta \varphi \cdot \nu=\frac{\partial^{2} \varphi}{\partial \nu^{2}} \cdot \nu$ on $\Gamma$ by applying (5.5) to $\varphi$ :

$$
\Delta \varphi \cdot \nu=\frac{\partial^{2} \varphi}{\partial \nu^{2}} \cdot \nu+\Delta_{\Gamma} \varphi \nu+\left(\frac{\partial \varphi}{\partial \nu}\right) \cdot \nu(\operatorname{div} \nu) \quad \text { on } \Gamma
$$

using (3.1c) (so that $\left.\frac{\partial^{2} \varphi}{\partial \tau_{i}^{2}}\right|_{\Gamma}=0$ and $\left.\Delta_{\Gamma} \varphi\right|_{\Gamma}=0$ ), and invoking further (3.1b) and (5.8) of Lemma 5.1, so that $\frac{\partial \varphi}{\partial \nu} \cdot \nu=0$ on $\Gamma$. Thus $(5.11 \mathrm{a}-\mathrm{b})$ is established from (5.12).

We reiterate that up to this point in our argument including Proposition 5.4, we have not used the over-determined B.C. (3.1d): $\left.\frac{\partial \varphi}{\partial \nu}\right|_{\Gamma}=0$; only the special property $\frac{\partial \varphi}{\partial \nu} \cdot \nu=0$ on $\Gamma$, as a consequence of $(3.1 \mathrm{~b}),(3.1 \mathrm{c})$ via Lemma 5.1. The assumption (3.1d) will be used for the first time in the next Step 4.

STEP 4.

Lemma 5.5. Assume (3.1b-d). Then

$$
\frac{\partial}{\partial \nu}\left(\frac{\partial \varphi}{\partial \nu}\right) \cdot \nu=0 \quad \text { on } \Gamma ; \quad \text { that is, } \quad \frac{\partial^{2} \varphi}{\partial \nu^{2}} \cdot \nu=0 \quad \text { on } \Gamma .
$$

Proof. By (3.1b) and (3.1c), Corollary 5.3 applies and thus we obtain $\left[\operatorname{div}\left(\frac{\partial \varphi}{\partial \nu}\right)\right]_{\Gamma}=0$ by $(5.10)$. Thus, at this point, involving also (3.1c), the following two properties hold true, under all present assumptions:

$$
\left.\frac{\partial \varphi}{\partial \nu}\right|_{\Gamma}=0,\left.\quad \operatorname{div}\left(\frac{\partial \varphi}{\partial \nu}\right)\right|_{\Gamma}=0
$$


Then the desired implication $(5.14) \Rightarrow(5.13)$ is merely an application of Lemma 5.1 with $w=\frac{\partial \varphi}{\partial \nu}$. In fact, in this notation, (5.14) is the assumption of Lemma 5.1 and (5.13) its conclusion.

SteP 5. We now return to the elliptic problem for $p$ in $(5.11 \mathrm{a}-\mathrm{b})$ and obtain, via (5.14) of Lemma 5.5, the following result.

THEOREM 5.6.

(i) The over-determined Stokes problem (3.1a-d) implies

$$
\begin{cases}\Delta p=0 & \text { on } \Omega \\ \left.\frac{\partial p}{\partial \nu}\right|_{\Gamma}=\Delta \varphi \cdot \nu=\frac{\partial^{2} \varphi}{\partial \nu^{2}} \cdot \nu=0 & \text { on } \Gamma .\end{cases}
$$

(ii) Hence,

$$
p \equiv \text { const } \quad \text { in } \Omega, \quad \nabla p \equiv 0 \quad \text { in } \Omega .
$$

Proof. (i) The $p$-problem (5.11a-b) of Proposition 5.4 specializes to the homogeneous problem $(5.15 \mathrm{a}-\mathrm{b})$, by application of (5.13) of Lemma 5.5 (which relies on the over-determined B.C. (3.1d)).

(ii) Green's first theorem applied to problem $(5.15 \mathrm{a}-\mathrm{b})$ yields

$$
0=\int_{\Omega} \Delta p p d \Omega=\int_{\Gamma} \frac{\partial p}{\partial \nu} p d \Gamma-\int_{\Omega}|\nabla p|^{2} d \Omega,
$$

and hence $\nabla p \equiv 0$ in $\Omega$.

STEP 6. Having obtained $\nabla p \equiv 0$ in (5.16), we return to problem (3.1a-d), which then becomes a standard over-determined eigenvalue/function problem for the Laplacian and thus yields a fortiori

$$
\varphi \equiv 0 \quad \text { in } \Omega,
$$

as noted in the proof of Theorem 1.D of Part I, in the paragraph below (1.12). [Over-determination, i.e., Cauchy data zero, on all of $\Gamma$ is too strong a condition at this stage.] Thus, Theorem 3.1 is proved.

REMARK 5.1. If we start instead with the Oseen problem (3.3), (3.4)in place of the Stokes equation (3.1a) - plus the additional conditions $(3.1 \mathrm{~b}-\mathrm{d})$ of Part II, we get the following elliptic problem:

$$
\left\{\begin{array}{l}
\Delta p=-\operatorname{div} L_{e}(\varphi) \text { in } \Omega \\
\left.\frac{\partial p}{\partial \nu}\right|_{\Gamma}=0
\end{array}\right.
$$


where

(5.20a) $\quad \operatorname{div} L_{e}(\varphi)=\operatorname{div}\left\{\left(y_{e} \cdot \nabla\right) \varphi+(\varphi \cdot \nabla) y_{e}\right\}$

$$
\begin{aligned}
& =2 \operatorname{div}\left[\left(y_{e} \cdot \nabla\right) \varphi\right]=2 \operatorname{div}\left[(\varphi \cdot \nabla) y_{e}\right] \\
& =2\left\{\left(\partial_{x_{1}} y_{e}\right) \cdot \nabla \varphi_{1}+\left(\partial_{x_{2}} y_{e}\right) \cdot \nabla \varphi_{2}+\left(\partial_{x_{3}} y_{e}\right) \cdot \nabla \varphi_{3}\right\} \\
& =2\left\{\left(\partial_{x_{1}} \varphi\right) \cdot \nabla y_{e 1}+\left(\partial_{x_{2}} \varphi\right) \cdot \nabla y_{e 2}+\left(\partial_{x_{3}} \varphi\right) \cdot \nabla y_{e 3}\right\}
\end{aligned}
$$

is actually a first-order differential operator. [The proof of $(3.20),(3.21)$ is given below and uses $\operatorname{div} \varphi \equiv 0$ and $\operatorname{div} y_{e} \equiv 0$ in $\Omega$.] At any rate, the pressure problem is no longer decoupled from $\varphi$ in the Oseen case in $(5.19 \mathrm{a}-\mathrm{b})$, unlike the Stokes case in $(5.15 \mathrm{a}-\mathrm{b})$. The above argument for the Stokes problem is no longer sufficient.

Proof of (3.20), (3.21). We shall treat both terms of $L_{e}(\varphi)$ in (3.4) separately.

STEP 1. We shall first show that $\operatorname{div} \varphi \equiv 0$ in $\Omega$ implies

$$
\begin{aligned}
\operatorname{div}\left[\left(y_{e} \cdot \nabla\right) \varphi\right]= & y_{e 1 x_{1}} \varphi_{1 x_{1}}+y_{e 2 x_{1}} \varphi_{1 x_{2}}+y_{e 3 x_{1}} \varphi_{1 x_{3}} \\
& +y_{e 1 x_{2}} \varphi_{2 x_{1}}+y_{e 2 x_{2}} \varphi_{2 x_{2}}+y_{e 3 x_{2}} \varphi_{2 x_{3}} \\
& +y_{e 1 x_{3}} \varphi_{3 x_{1}}+y_{e 2 x_{3}} \varphi_{3 x_{2}}+y_{e 3 x_{3}} \varphi_{3 x_{3}} .
\end{aligned}
$$

The above identity displays symmetry between $y_{e}$ and $\varphi$. Thus, it can either be read horizontally, leading to

$$
\begin{aligned}
& =\partial_{x_{1}}\left[\begin{array}{l}
y_{e 1} \\
y_{e 2} \\
y_{e 3}
\end{array}\right] \cdot\left[\begin{array}{l}
\varphi_{1 x_{1}} \\
\varphi_{1 x_{2}} \\
\varphi_{1 x_{3}}
\end{array}\right]+\partial_{x_{2}}\left[\begin{array}{l}
y_{e 1} \\
y_{e 2} \\
y_{e 3}
\end{array}\right] \cdot\left[\begin{array}{l}
\varphi_{2 x_{1}} \\
\varphi_{2 x_{2}} \\
\varphi_{2 x_{3}}
\end{array}\right]+\partial_{x_{3}}\left[\begin{array}{l}
y_{e 1} \\
y_{e 2} \\
y_{e 3}
\end{array}\right] \cdot\left[\begin{array}{l}
\varphi_{3 x_{1}} \\
\varphi_{3 x_{2}} \\
\varphi_{3 x_{3}}
\end{array}\right] \\
& =\left(\partial_{x_{1}} y_{e}\right) \cdot \nabla \varphi_{1}+\left(\partial_{x_{2}} y_{e}\right) \cdot \nabla \varphi_{2}+\left(\partial_{x_{3}} y_{e}\right) \cdot \nabla \varphi_{3},
\end{aligned}
$$

or vertically, leading to

$$
\begin{aligned}
& =\partial_{x_{1}}\left[\begin{array}{l}
\varphi_{1} \\
\varphi_{2} \\
\varphi_{3}
\end{array}\right] \cdot\left[\begin{array}{l}
y_{e 1 x_{1}} \\
y_{e 1 x_{2}} \\
y_{e 1 x_{3}}
\end{array}\right]+\partial_{x_{2}}\left[\begin{array}{l}
\varphi_{1} \\
\varphi_{2} \\
\varphi_{3}
\end{array}\right] \cdot\left[\begin{array}{l}
y_{e 2 x_{1}} \\
y_{e 2 x_{2}} \\
y_{e 2 x_{3}}
\end{array}\right]+\partial_{x_{3}}\left[\begin{array}{c}
\varphi_{1} \\
\varphi_{2} \\
\varphi_{3}
\end{array}\right] \cdot\left[\begin{array}{l}
y_{e 3 x_{1}} \\
y_{e 3 x_{2}} \\
y_{e 3 x_{3}}
\end{array}\right] \\
& =\left(\partial_{x_{1}} \varphi\right) \cdot \nabla_{y_{e 1}}+\left(\partial_{x_{2}} \varphi\right) \cdot \nabla y_{e 2}+\left(\partial_{x_{3}} \varphi\right) \cdot \nabla y_{e 3} .
\end{aligned}
$$

To prove $(5.22)$, we compute 


$$
\begin{aligned}
& \operatorname{div}\left[\left(y_{e} \cdot \nabla\right) \varphi\right] \\
& =\operatorname{div}\left[\begin{array}{c}
y_{e} \cdot \nabla \varphi_{1} \\
y_{e} \cdot \nabla \varphi_{2} \\
y_{e} \cdot \nabla \varphi_{3}
\end{array}\right]=\sum_{i=1}^{3} \partial_{x_{i}}\left(y_{e 1} \varphi_{i x_{1}}+y_{e 2} \varphi_{i x_{2}}+y_{e 3} \varphi_{i x_{3}}\right) \\
& =y_{e 1 x_{1}} \varphi_{1 x_{1}}+y_{e 1} \varphi 1 x_{1} x_{1}+y_{e 2 x_{1}} \varphi_{1 x_{2}}+y_{e 2} \varphi_{1 x_{2} x_{1}} \\
& +y_{e 3 x_{1}} \varphi_{1 x_{3}}+y_{e .3 \varphi 1 x_{3} x_{1}} \\
& +y_{e 1 x_{2}} \varphi_{2 x_{1}}+y_{e 1} \varphi_{2 x_{1} x_{2}}+y_{e 2 x_{2}} \varphi_{2 x_{2}}+y_{e 2} \varphi_{2 x_{2} x_{2}} \\
& +y_{e 3 x_{2}} \varphi_{2 x_{3}}+y_{e 3 x_{3} x_{2}} \\
& +y_{e 1 x_{3}} \varphi_{3 x_{1}}+y_{e 1} \varphi 3 x_{1} x_{3}+y_{e 2 x_{3}} \varphi_{3 x_{2}}+y_{e 2} \varphi_{3 x_{2} x_{3}} \\
& +y_{e 3 x_{3}} \varphi_{3 x_{3}}+y_{\text {e.3 }} .
\end{aligned}
$$

But, from (3.1b): $\operatorname{div} \varphi=\varphi_{1 x_{1}}+\varphi_{2 x_{2}}+\varphi_{3 x_{3}} \equiv 0$ in $\Omega$, we obtain for the terms of the second column, fourth column, sixth column in (5.25):

$$
\begin{array}{ll}
(5.26 \mathrm{a}) & y_{e 1}\left(\varphi_{1 x_{1} x_{1}}+\varphi_{2 x_{1} x_{2}}+\varphi_{3 x_{1} x_{3}}\right)=y_{e 1} \partial_{x_{1}}(\operatorname{div} \varphi) \equiv 0 ; \\
(5.26 \mathrm{~b}) & y_{e 2}\left(\varphi_{1 x_{2} x_{1}}+\varphi_{2 x_{2} x_{2}}+\varphi_{3 x_{2} x_{3}}\right)=y_{e 2} \partial_{x_{2}}(\operatorname{div} \varphi) \equiv 0 ; \\
(5.26 \mathrm{c}) & y_{e 3}\left(\varphi_{1 x_{3} x_{1}}+\varphi_{2 x_{3} x_{2}}+\varphi_{3 x_{3} x_{3}}\right)=y_{e 3} \partial_{x_{3}}(\operatorname{div} \varphi) \equiv 0 .
\end{array}
$$

Using $(5.26 \mathrm{a}-\mathrm{c})$ in $(5.25)$ yields $(5.22)$.

STEP 2. By the symmetry of (5.22) in $y_{e}$ and $\varphi$, it follows that the condition $\operatorname{div} y_{e} \equiv 0$ in $\Omega$ likewise implies the RHS of (5.22):

$$
\operatorname{div}\left[(\varphi \cdot \nabla) y_{e}\right]=\text { RHS of }(5.22) \text {. }
$$

STEP 3. Thus, under both conditions $(3.1 \mathrm{~b})$ for $\varphi$ and $(3.5 \mathrm{~b})$ for $y_{e}$, we obtain

$$
\begin{aligned}
\operatorname{div} \varphi \equiv 0, \operatorname{div} y_{e} \equiv 0 \text { in } \Omega & \\
\Rightarrow \operatorname{div}\left[\left(y_{e} \cdot \nabla\right) \varphi\right] & \equiv \operatorname{div}\left[(\varphi \cdot \nabla) y_{e}\right] \equiv \operatorname{RHS} \text { of }(5.22) \\
& =(5.23)=(5.24),
\end{aligned}
$$

and so (5.20), (5.21) follow from (5.28).

REMARK 5.2. We remark that if the Oseen equation $(3.3),(3.4)$ is replaced by

$$
(-\Delta) \varphi+\left(y_{e} \cdot \nabla\right) \varphi-(\varphi \cdot \nabla) y_{e}+\nabla p=\lambda \varphi
$$

i.e., with the minus sign between the two first-order terms rather than the plus sign as in (3.4), then one would still get $(5.15 \mathrm{a}-\mathrm{b}),(5.16)$, and the unique continuation conclusion of Theorem 3.1 of Part II would still hold. 
We conclude this section by providing the projection of the Oseen equation (3.3) onto the space $H$ [C-F, p. 7], [Te, p. 15], where $L_{2}(\Omega)=H+H^{\perp}$ (orthogonal sum) with

$$
\begin{aligned}
\text { (5.29a) } \quad H & =\left\{h \in\left(L_{2}(\Omega)\right)^{d}: \operatorname{div} h \equiv 0 \text { in } \Omega ; h \cdot \nu=0 \text { on } \Gamma\right\} ; \\
\text { (5.29b) } \quad H^{\perp} & =\left\{u \in\left(L_{2}(\Omega)\right)^{d}: u=\nabla p, p \in H^{1}(\Omega)\right\} .
\end{aligned}
$$

COROLlaRY 5.7. With reference to the over-determined Oseen problem (3.3), (3.4), (3.1b-d) with $\varphi \in\left(H^{2}(\Omega)\right)^{d}, p \in H^{1}(\Omega)$, we have

(5.30a) $\varphi, \Delta \varphi \in H \equiv\left\{f \in\left(L_{2}(\Omega)\right)^{d}: \operatorname{div} f \equiv 0\right.$ in $\Omega ; f \cdot \nu=0$ on $\left.\Gamma\right\}$.

Hence, if $P$ is the Helmholtz/Leray projector [C-F, p. 9] $\left(L_{2}(\Omega)\right)^{d} \rightarrow H$, then

$$
P \varphi=\varphi ; \quad P \Delta \varphi \equiv \Delta \varphi ; \quad P \nabla p=0,
$$

and applying $P$ across the Oseen equation (3.3) yields

$$
(-\Delta) \varphi+P L_{e}(\varphi)=\lambda \varphi \quad \text { in } \Omega .
$$

Proof. Under the present assumptions, the critical relation $\Delta \varphi \cdot \nu=0$ on $\Gamma$ was established in (5.13), (5.15b). Then, via (5.29a-b), (5.30a-b), application of $P$ to (1.3) yields (3.31).

The p-problem $(5.19 a-b)$. Unlike the Stokes problem, the $p$-problem $(5.19 \mathrm{a}-\mathrm{b})$ is coupled in the Oseen case.

Proposition 5.8. With reference to the p-problem $(5.19 \mathrm{a}-\mathrm{b})$, we have

where

$$
\int_{\Omega}|\nabla p|^{2} d \Omega=-\left(L_{e}(\varphi), \nabla p\right)_{\Omega},
$$

Hence,

$$
\left(L_{e}(\varphi), \nabla p\right)_{\Omega}=2\left(\left(y_{e} \cdot \nabla\right) \varphi, \nabla p\right)_{\Omega}=2\left((\varphi \cdot \nabla) y_{e}, \nabla p\right)_{\Omega}
$$

Proof. (5.32) follows by first taking the (complex) $\left(L_{2}(\Omega)\right)^{d}$-inner product of (5.19a) with $\bar{p}$ and applying Green's first theorem using (5.19b):

$$
\int_{\Omega} \Delta p \bar{p} d \Omega=\int_{\Gamma} \frac{\partial p}{\partial \nu} \bar{p} d \Gamma-\int_{\Omega}|\nabla p|^{2} d \Omega .
$$

Moreover, since $\left.L_{2}(\varphi)\right|_{\Gamma}=0$ by (3.4) via (3.5c), (3.1c), via the divergence formula we have

$$
-\left(\operatorname{div} L_{e}(\varphi), p\right)_{\Omega}=-\int_{\Gamma} \bar{p} L_{e}(\varphi) \cdot \forall \partial \Gamma+\left(L_{e}(\varphi), \nabla p\right) .
$$

Equating (5.36) and (5.37) —as required by (5.19a)—yields (5.32). 
To prove (5.33), we either recall (5.20a-b) as applied to (5.37) and repeat the argument in (5.37):

$$
\begin{aligned}
\text { (5.38) LHS of }(5.37) & =-2\left(\operatorname{div}\left[\left(y_{e} \cdot \nabla\right) \varphi\right], p\right) \\
& =-2 \int_{\Gamma} \bar{p}\left(y_{e} \cdot \nabla\right) \varphi \bar{\nu} \Gamma+2\left(\left(y_{e} \cdot \nabla\right) \varphi, \nabla p\right)_{\Omega}, \\
\text { (5.39) LHS of }(5.37) & =-2\left(\operatorname{div}\left[(\varphi \cdot \nabla) y_{e}\right], p\right) \\
& =-2 \int_{\Gamma} \bar{p}(\varphi \cdot \nabla) y_{e} \downarrow d \Gamma+2\left((\varphi \cdot \nabla) y_{e}, \nabla p\right)_{\Omega},
\end{aligned}
$$

or else, equivalently, recalling (3.4), we obtain

$$
\begin{aligned}
& L_{e}(\varphi)=\left[\left(y_{e} \cdot \nabla\right) \varphi-(\varphi \cdot \nabla) y_{e}\right]+2(\varphi \cdot \nabla) y_{e}, \\
& L_{e}(\varphi)=\left[(\varphi \cdot \nabla) y_{e}-\left(y_{e} \cdot \nabla\right) \varphi\right]+2\left(y_{e} \cdot \nabla\right) \varphi,
\end{aligned}
$$

where

$$
\left[\left(y_{e} \cdot \nabla\right) \varphi-(\varphi \cdot \nabla) y_{e}\right] \in H ;\left[(\varphi \cdot \nabla) y_{e}-\left(y_{e} \cdot \nabla\right) \varphi\right] \in H,
$$

since these two terms are both divergence-free by (5.28), and both vanish on $\Gamma$, by (3.1c), (3.5c). Hence,

$$
\left(\left[\left(y_{e} \cdot \nabla\right) \varphi-(\varphi \cdot \nabla) y_{e}\right], \nabla p\right)_{\Omega}=0, \quad\left(\left[(\varphi \cdot \nabla) y_{e}-\left(y_{e} \cdot \nabla\right) \varphi\right], \nabla p\right)_{\Omega}=0,
$$

as $\nabla p \in H^{\perp}$. Then (5.40), (5.41), (5.43) yield again (5.33).

Finally, (5.32), (5.33) yield (5.34), (5.35) via the Schwarz inequality.

REMARK 5.3. The author has a few proofs showing that at least for $y_{e}$ sufficiently small in the $\left(W^{1, \infty}(\Omega)\right)^{d}$-norm, the Oseen problem (3.3), (3.1b), (3.1c), (3.1d) implies

$$
\varphi \equiv 0 \quad \text { in } \Omega, \text { hence } \nabla p \equiv 0 \quad \text { in } \Omega .
$$

(Notice the reverse order: First $\varphi \equiv 0$ in $\Omega$, then $\nabla p \equiv 0$ in $\Omega$, via (3.3); in the above Stokes argument, we obtained first $\nabla p \equiv 0$ in $\Omega$, and then $\varphi \equiv 0$ in $\Omega$ via (1.1a).) One such proof uses the Rellich multiplier $\left(\left(x-x_{0}\right) \cdot \nabla\right) \varphi$; another, a Carleman inequality. They will be given elsewhere [T.4].

Instead, in the next section we provide a topological result complementing the conclusion given by (5.44).

II.6. The set of "good" equilibrium solutions $y_{e}$ is open in $\left(W^{1, \infty}(\Omega)\right)^{d}$ for the Oseen problem

The given Oseen eigenproblem. In this section we consider the eigenvalue/function problem for the Oseen equation (3.3), (3.4). Thus let $y_{e} \in$ $\left(H^{2}(\Omega)\right)^{d} \cap V$ be a given equilibrium solution. For such $y_{e}$, let

$$
\lambda \in \mathbb{C}, \quad w \in\left(H^{2}(\Omega)\right)^{d}, \quad p \in H^{1}(\Omega),
$$


with, say, $w$ normalized in $\left(L_{2}(\Omega)\right)^{d}$, satisfy the following Oseen eigenproblem:

$$
\begin{cases}(-\Delta) w+L_{e}(w)+\nabla p=\lambda w & \text { in } \Omega ; \\ \operatorname{div} w \equiv 0 & \text { in } \Omega ; \\ w=0 & \text { on } \Gamma ;\end{cases}
$$

so that $\lambda$ is an eigenvalue and $w$ a (generally speaking, complex-valued) corresponding eigenfunction.

Applying the Helmholtz-Leray projector $P$ to $(6.2 \mathrm{a})$ we obtain the corresponding pressure-free formulation

$$
\begin{gathered}
\begin{cases}(-P \Delta) w+P L_{e}(w)=\lambda w & \text { in } \Omega ; \\
\operatorname{div} w \equiv 0 & \text { in } \Omega ; \text { or } \quad \mathcal{A}_{e} w=\lambda w ; \\
w=0 & \text { on } \Gamma ;\end{cases} \\
\left\{\begin{array}{l}
\mathcal{A}_{e}=A+A_{e}: H \supset \mathcal{D}\left(\mathcal{A}_{e}\right)=\mathcal{D}(A)=\left(H^{2}(\Omega)\right)^{d} \cap V \rightarrow H ; \\
\mathcal{A}_{e} f=-P \Delta f+P L_{e}(f), f \in \mathcal{D}\left(\mathcal{A}_{e}\right) ;
\end{array}\right. \\
A f=-P \Delta f: H \supset \mathcal{D}(A) \rightarrow H ; A_{e} f=P L_{e}(f) ; \mathcal{D}\left(A_{e}\right)=V=\mathcal{D}\left(A^{1 / 2}\right) .
\end{gathered}
$$

As is well known, $A$ is a self-adjoint positive definite operator in $H$ with compact (resolvent) $A^{-1}$ on $H$ [C-F, p. 32]. Moreover, the (closed) operator $-\mathcal{A}_{e}$ likewise has compact resolvent and generates a s.c. analytic semigroup on $H$ ( $-\mathcal{A}_{e}$ is, in fact, sectorial). It follows that the (closed) operator $-\mathcal{A}_{e}$ has a finite number $N$ of unstable eigenvalues (consistently with (6.2a), these will be denoted by $\left\{-\lambda_{j}\right\}_{j=1}^{\infty}$ ), with $\operatorname{Re}\left(-\lambda_{j}\right) \geq 0$ (the unstable eigenvalues of the free dynamic generator $\left.-\mathcal{A}_{e}\right)$ :

$$
\operatorname{Re}\left(-\lambda_{N+1}\right)<0 \leq \operatorname{Re}\left(-\lambda_{N}\right) \leq \cdots \leq \operatorname{Re}\left(-\lambda_{1}\right) ; \quad\left\{\lambda_{j}\right\}_{j=1}^{\infty} \subset \sigma_{p}\left(\mathcal{A}_{e}\right) .
$$

The eigenvalues are repeated according to their algebraic multiplicity $\ell_{j}$.

$A$ small perturbation of $(6.2 a-c)$. Let $y_{e} \in\left(H^{2}(\Omega)\right)^{d} \cap V$ and let

$$
\mathcal{B}_{y_{e}}(R)=\left\{\tilde{y}_{e} \in\left(H^{2}(\Omega)\right)^{d} \cap V:\left\|\tilde{y}_{e}-y_{e}\right\|_{\left(W^{1, \infty}(\Omega)\right)^{d}}<R\right\}
$$

be a ball of equilibrium solutions centered at $y_{e}$ of radius $R$, where the norm of $\left(W^{1, \infty}(\Omega)\right)^{d}$ is defined by

$$
\|f\|_{\left(W^{1, \infty}(\Omega)\right)^{d}}=\|f\|_{\left(L_{\infty}(\Omega)\right)^{d}}+\|\nabla f\|_{\left(L_{\infty}(\Omega)\right)^{d}} .
$$

THEOREM 6.1. With reference to the eigenproblem $(6.2 \mathrm{a}-\mathrm{c})$ or $(6.4 \mathrm{a}-\mathrm{c})$ in $\{\lambda, w\}$ generated by the equilibrium solution $y_{e}$, assume that

$$
\left.\frac{\partial w}{\partial \nu}\right|_{\Gamma} \not \equiv 0
$$


Then there exists a sufficiently small $R>0$ such that for every equilibrium solution $\tilde{y}_{e} \in \mathcal{B}_{y_{e}}(R)$ defined in (6.8a), one has the following Oseen eigenproblem in $\{\tilde{\lambda}, \tilde{w}\}$ :

$$
\begin{cases}(-\Delta) \tilde{w}+L_{\tilde{e}}(\tilde{w})+\nabla \tilde{p}=\tilde{\lambda} \tilde{w} & \text { in } \Omega ; \\ \operatorname{div} \tilde{w} \equiv 0 & \text { in } \Omega ; \quad \mathcal{A}_{\tilde{e}} \tilde{w}=\tilde{\lambda} w \\ \tilde{w}=0 & \text { on } \Gamma ;\end{cases}
$$

for some $\tilde{p} \in H^{1}(\Omega)$, with the following properties:

(i) $\tilde{\lambda}$ has the same algebraic multiplicity of $\lambda$;

(ii) we have

$$
\|\tilde{w}-w\|_{\left(L_{2}(\Omega)\right)^{d}} \leq C\left\|\tilde{y}_{e}-y_{e}\right\|_{\left(W^{1, \infty}(\Omega)\right)^{d}}
$$

(iii) in particular,

$$
\left\|\frac{\partial \hat{w}}{\partial \nu}-\frac{\partial w}{\partial \nu}\right\|_{\left(H^{-3 / 2}(\Gamma)\right)^{d}} \leq C\left\|\tilde{y}_{e}-y_{e}\right\|_{\left(W^{1, \infty}(\Omega)\right)^{d}},
$$

so that, in particular,

$$
\left.\frac{\partial \tilde{w}}{\partial \nu}\right|_{\Gamma} \not \equiv 0
$$

Proof. This result follows as an application of [Ka, Thm. 3.16, in particular, Thm. 3.18, pp. 212-214], in the following way. We take as closed operator $T$ in [Ka] the operator $T=\mathcal{A}_{e}=A+A_{e}$ in (6.5), with perturbation (called $A$ in $\left[\mathrm{Ka}\right.$, Thm. 3.18]) given by $A_{\tilde{e}}-A_{e}$, so that the perturbed operator, called $S$ in $[\mathrm{Ka}]$, is

$$
S=\mathcal{A}_{e}+\left(A_{\tilde{e}}-A_{e}\right)=\left(A+A_{e}\right)+\left(A_{\tilde{e}}-A_{e}\right)=A+A_{\tilde{e}}=\mathcal{A}_{\tilde{e}},
$$

as desired. The perturbation $A_{\tilde{e}}-A_{e}$ is $A$-bounded, hence $\mathcal{A}_{e}$-bounded; in fact, $A_{\tilde{e}}-A_{e}$ is $A^{1 / 2}$-bounded. More precisely, in view of (6.5), (6.6), we estimate, for $f \in\left(H^{2}(\Omega)\right)^{d} \cap V=\mathcal{D}\left(\mathcal{A}_{e}\right)=\mathcal{D}\left(\mathcal{A}_{\tilde{e}}\right)$, via $(6.3)$, (6.4):

$$
\begin{aligned}
\left\|\left(\mathcal{A}_{\tilde{e}}-\mathcal{A}_{e}\right) f\right\|_{H} & =\left\|P\left(L_{\tilde{e}}-L_{e}\right)(f)\right\|_{H} \\
& \leq\|P\|\left\|\left(\left(y_{\tilde{e}}-y_{e}\right) \cdot \nabla\right) f+(f \cdot \nabla)\left(y_{\tilde{e}}-y_{e}\right)\right\| \\
& \leq C\left\|y_{\tilde{e}}-y_{e}\right\|_{\left(W^{1, \infty}(\Omega)\right)^{d}}\left[\|f\|_{H}+\|f\|_{V}\right] .
\end{aligned}
$$

Thus, in the condition [Ka, Eqn. (3.14), p. 214],

$$
\sup _{\zeta \in \Gamma}\left[a\left\|R\left(\zeta, \mathcal{A}_{e}\right)\right\|+b\left\|\mathcal{A}_{e} R\left(\zeta, \mathcal{A}_{e}\right)\right\|\right]<1,
$$

which is required for the application of [Ka, Thm. 3.18, p. 214], we can take both constants $a=b=C\left\|y_{\tilde{e}}-y_{e}\right\|_{\left(W^{1, \infty}(\Omega)\right)^{d}}$ to be arbitrarily small, by taking $\left\|y_{\tilde{e}}-y_{e}\right\|_{\left(W^{1, \infty}(\Omega)\right)^{d}}$ sufficiently small, via (6.18), no matter what closed 
path $\Gamma$ surrounding the eigenvalue $\lambda$ (or any finite number of eigenvalues) one considers. This is so also because $\left\|\mathcal{A}_{e} R\left(\zeta,-\mathcal{A}_{e}\right)\right\|$ is uniformly bounded by a constant, for all $\zeta \in \varrho\left(-\mathcal{A}_{e}\right)$ (the resolvent set of $\left.-\mathcal{A}_{e}\right)$, since $-\mathcal{A}_{e}$ is the generator of a s.c. analytic semigroup on $H$. Thus, [Ka, Thm. 3.18, p. 214] is applicable and produces conclusions (i) and (ii), from which conclusion (iii) $=(6.13)$ follows. Because of (6.9), (6.14) then follows as well, for sufficiently small $a=b$.

A generalization to finitely many eigenvalues. Indeed, as noted at the end of the proof of Theorem 6.1 given above, [Ka, Thm. 3.16; in particular, Thm. 3.18; pp. 212-214] permits us to obtain a more general perturbation result involving finitely many eigenvalues, say, the unstable eigenvalues $-\lambda_{1}, \ldots,-\lambda_{N}$ of $-\mathcal{A}_{e}$ in (6.7). To this end, we generalize problem $(6.2 \mathrm{a}-\mathrm{c})$ [or $(6.4 \mathrm{a}-\mathrm{c})]$ by considering for $j=1, \ldots, N$ :

$$
\begin{cases}(-\Delta) w_{j}+L_{e}\left(w_{j}\right)+\nabla p_{j}=\lambda_{j} w_{j} & \text { in } \Omega ; \\ \operatorname{div} w_{j} \equiv 0 & \text { in } \Omega ; \\ w_{j}=0 & \text { on } \Gamma ;\end{cases}
$$

or

$$
\left\{\begin{array}{l}
(-P \Delta) w_{j}+P L_{e}\left(w_{j}\right)=\lambda_{j} w_{j} ; \\
\operatorname{div} w_{j} \equiv 0, \\
w_{j}=0 .
\end{array} \quad \text { or } \quad \mathcal{A}_{e} w_{j}=\lambda_{j} w_{j} ;\right.
$$

TheOREM 6.2. With reference to the eigenvalue problems $(6.20 \mathrm{a}-\mathrm{c})$ or $(6.21 \mathrm{a}-\mathrm{c})$ in $\left\{\lambda_{j}, w_{j}\right\}$ generated by the equilibrium solution $y_{e}$, assume that

$$
\left.\frac{\partial w_{j}}{\partial \nu}\right|_{\Gamma} \not \equiv 0, \quad j=1, \ldots, N .
$$

Then there exists a sufficiently small $R$ such that for every equilibrium solution $\tilde{y}_{e} \in \mathcal{B}_{y_{e}}(R)$ defined by (6.8a), one obtains the following Oseen eigenproblem in $\left\{\tilde{\lambda}_{j}, \tilde{w}_{j}\right\}$ :

$$
\begin{cases}(-\Delta) \tilde{w}_{j}+L_{\tilde{e}}\left(\tilde{w}_{j}\right)+\nabla \tilde{p}_{j}=\tilde{\lambda}_{j} \tilde{w}_{j} & \text { in } \Omega ; \\ \operatorname{div} \tilde{w}_{j} \equiv 0 & \text { in } \Omega ; \quad \mathcal{A}_{\tilde{e}} \tilde{w}_{j}=\tilde{\lambda}_{j} \tilde{w}_{j} \\ \tilde{w}_{j}=0 & \text { on } \Gamma,\end{cases}
$$

$L_{\tilde{e}}$ defined by (6.11), for some $\tilde{p}_{j} \in H^{1}(\Omega)$, with the following properties:

(i) each $\tilde{\lambda}_{j}$ has the same algebraic multiplicity as $\lambda_{j}$;

(ii) the dimension of the (unstable) eigenspace generated by the span of the generalized eigenfunctions corresponding to $\tilde{\lambda}_{1}, \ldots, \tilde{\lambda}_{N}$ is the same as the dimension of the (unstable) eigenspace generated by the span of the generalized eigenfunctions corresponding to $\lambda_{1}, \ldots, \lambda_{N}$; 
(iii) we have

$$
\sum_{j=1}^{N}\left\|\tilde{w}_{j}-w_{j}\right\|_{\left(L_{2}(\Omega)\right)^{d}} \leq C\left\|\tilde{y}_{e}-y_{e}\right\|_{\left(W^{1, \infty}(\Omega)\right)^{d}} ;
$$

(iv) in particular,

$$
\sum_{j=1}^{N}\left\|\frac{\partial \tilde{w}_{j}}{\partial \nu}-\frac{\partial w_{j}}{\partial \nu}\right\|_{\left(H^{-3 / 2}(\Gamma)\right)^{d}} \leq C\left\|\tilde{y}_{e}-y_{e}\right\|_{\left(W^{1, \infty}(\Omega)\right)^{d}}
$$

so that, in particular,

$$
\left.\frac{\partial \tilde{w}_{j}}{\partial \nu}\right|_{\Gamma} \not \equiv 0, \quad j=1, \ldots, N
$$

Next, call an equilibrium solution $y_{e}$ good if the over-determined Oseen problem (3.3), (3.4), (3.1b-d):

$$
\begin{cases}(-\Delta) \varphi+L_{e}(\varphi)+\nabla p=\lambda \varphi & \text { in } \Omega ; \\ \operatorname{div} \varphi \equiv 0 & \text { in } \Omega ; \\ \left.\varphi\right|_{\Gamma} \equiv 0 ;\left.\quad \frac{\partial \varphi}{\partial \nu}\right|_{\Gamma} \equiv 0, & \end{cases}
$$

implies the (desired) conclusion of Theorem 3.1 (for the Stokes problem)

$$
\varphi \equiv 0 \quad \text { in } \Omega ; \quad p \equiv \text { const } \quad \text { in } \Omega .
$$

Then Theorem 6.1, or Theorem 6.2, can be reformulated as follows via a contradiction argument.

THEOREM 6.3.

(a) The set of good equilibrium solutions $y_{e}$ is open in the $\left(W^{1, \infty}(\Omega)\right)^{d}$ norm.

(b) In particular, due to Theorem 3.1, the following holds true: there is a sphere $\mathcal{B}_{y_{e}=0}(R)$ of suitably small radius $R>0$ around the zero equilibrium solution $y_{e}=0$ (Stokes problem) such that every $\tilde{y}_{e} \in \mathcal{B}_{y_{e}=0}(R)$ is a good equilibrium solution; that is, the implication $(6.27 \mathrm{a}-\mathrm{c}) \Rightarrow(6.28)$ holds true for all such $\tilde{y}_{e}$ of sufficiently small $\left(W^{1, \infty}(\Omega)\right)^{d}$-norm.

Corollary 6.4. In the Oseen eigenvalue problem consisting of (4.2a) with the additional term $L_{e}\left(w_{j}\right)$, i.e., of

$$
(-\Delta) w_{j}+L_{e}\left(w_{j}\right)+\nabla p_{j}=\lambda_{j} w_{j} \quad \text { in } \Omega,
$$

along with conditions $(4.2 \mathrm{a}-\mathrm{c})$, the linear independence of the boundary traces $\left\{\left.\partial_{\nu} w_{i j}\right|_{\Gamma}\right\}_{j=1}^{\ell_{i}}$ in $\left(L_{2}(\Gamma)\right)^{d}$, as in conclusion (4.3) of Theorem 4.1, is still valid for the equilibrium solutions $y_{e}$ for which Theorem 6.3 holds true. 
REMARK 6.1. While the unique continuation for the over-determined Oseen problem $(6.27 \mathrm{a}-\mathrm{c})$ is of interest for any potential eigenvalue $\lambda$ of the operator $\mathcal{A}_{e}$ in $(6.5 \mathrm{a}-\mathrm{b})$, thus typically with $\operatorname{Re} \lambda>0$, it is the case $\operatorname{Re} \lambda \leq 0$ that corresponds to the unstable eigenvalues of the free dynamics generator $-\mathcal{A}_{e}$ (see (6.7)) that is critical in stabilization problems [B-L-T.1].

II.7. Proof of Theorem 3.2. In this section, we provide a proof of Theorem 3.2, which is based on [B-L-T.1, Sect. 3.6]. We return to the Oseen problem $(3.7 \mathrm{a}-\mathrm{c})$. Following a classical elliptic approach, we extend $\varphi \in\left(H^{2}(\Omega)\right)^{d}$ and $p \in H^{1}(\Omega)$ by zero locally across the portion $\Gamma_{1}$ of the boundary - where $(3.7 \mathrm{c})$ holds true - onto a set $\omega$ exterior to $\Omega$. Thus, we set

$$
\hat{\varphi}=\left\{\begin{array}{ll}
\varphi & \text { in } \Omega ; \\
0 & \text { in } \omega ;
\end{array} \quad \hat{p}=\left\{\begin{array}{ll}
p & \text { in } \Omega ; \\
0 & \text { in } \omega ;
\end{array} \quad G=\Omega \cup \omega .\right.\right.
$$

Let $\hat{y}_{e}$ be a smooth extension of $y_{e}$ onto $\omega$ so that $\hat{y}_{e} \in H(G)$. Denote by $L_{\hat{e}}$ the operator (3.4) with $y_{e}$ replaced by $\hat{y}_{e}$.

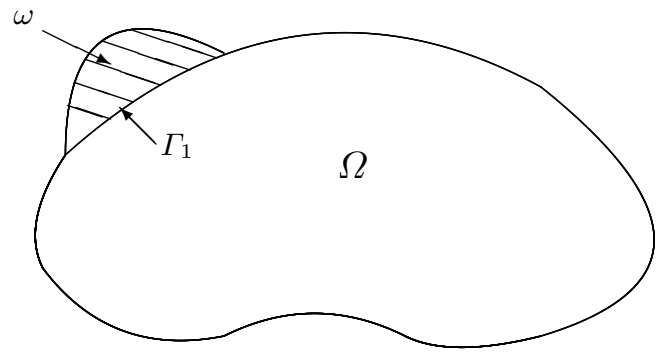

SteP 1. We claim that the extended pair $\{\hat{\varphi}, \hat{p}\}$ satisfies, in the distributional sense, over $G=\Omega \cup \omega$,

$$
(-\Delta) \hat{\varphi}+L_{\hat{e}}(\hat{\varphi})+\nabla \hat{p}-\lambda \hat{\varphi}= \begin{cases}(-\Delta) \varphi+L_{e}(\varphi)+\nabla p-\lambda \varphi & \text { in } \Omega ; \\ 0 & \text { in } \omega ;\end{cases}
$$

that is, for all $\psi \in\left(C_{0}^{\infty}(G)\right)^{d}$, we have

$$
\begin{aligned}
\int_{G}\left[(-\Delta) \hat{\varphi}+L_{\hat{e}}(\hat{\varphi})+\nabla \hat{p}-\lambda \hat{\varphi}\right] \cdot \psi d G \\
=\int_{\Omega}\left[(-\Delta) \varphi+L_{e}(\varphi)+\nabla p-\lambda \varphi\right] \cdot \psi d \Omega .
\end{aligned}
$$

To this end, in the following identities, we use the definition of distributional derivative, followed by the definition of $\{\hat{\varphi}, \hat{p}\}$ in (7.1), and usual Green identities on $\Omega$, with sufficiently smooth boundary $\Gamma$, and $\varphi \in\left(H^{2}(\Omega)\right)^{d}$, 
$p \in H^{1}(\Omega), \psi \in\left(C_{0}^{\infty}(G)\right)^{d}$. We obtain

$$
\begin{aligned}
\int_{G}(-\Delta) \hat{\varphi} \cdot \psi d G & =-\int_{G} \hat{\varphi} \cdot \Delta \psi d G=-\int_{\Omega} \varphi \cdot \Delta \psi d \Omega \\
& =\int_{\Omega}(-\Delta) \varphi \cdot \psi d \Omega-\int_{\Gamma} \varphi \cdot \frac{\partial \psi}{\partial \nu} d \Gamma+\int_{\Gamma} \frac{\partial \varphi}{\partial \nu} \cdot \psi d \Gamma,
\end{aligned}
$$

by use of Green's second theorem in passing from (7.4) to (7.5). Moreover

$$
\begin{aligned}
\int_{G} \nabla \hat{p} \cdot \psi d G & =-\int_{G} \hat{p} \operatorname{div} \psi d G=-\int_{\Omega} p \operatorname{div} \psi d \Omega \\
& =\int_{\Gamma} p \psi \cdot \nu d \Gamma+\int_{\Omega} \nabla p \cdot \psi d \Omega,
\end{aligned}
$$

by use of the divergence formula in passing from (7.6) to (7.7). Summing up (7.5) and (7.7) yields

$$
\begin{aligned}
\int_{G}[(-\Delta) \hat{\varphi}+\nabla \hat{p}] \cdot \psi d G=\int_{\Omega} & {[(-\Delta) \varphi+\nabla p] \cdot \psi d \Omega+\int_{\Gamma} \frac{\partial \cdot \frac{\partial \psi}{\partial \nu} d \Gamma}{} d \Gamma } \\
& +\int_{\Gamma}\left[\frac{\partial \varphi}{\partial \nu}-p \nu\right] \cdot \psi d \Gamma .
\end{aligned}
$$

The two boundary integrals vanish since $\left.\psi\right|_{\Gamma \backslash \Gamma_{1}}=0,\left.\frac{\partial \psi}{\partial \nu}\right|_{\Gamma \backslash \Gamma_{1}}=0$ as $\psi \in$ $\left(C_{0}^{\infty}(G)\right)^{d}$, while $\left.\varphi\right|_{\Gamma_{1}}=0$ and $\left[\frac{\partial \varphi}{\partial \nu}-p \nu\right]_{\Gamma_{1}}=0$ by $(3.7 \mathrm{c})$.

By the definition of $L_{e}$ in (3.4), one readily obtains via (7.1) for $\hat{\varphi}$

$$
\begin{aligned}
\int_{G} L_{\hat{e}}(\hat{\varphi}) \cdot \psi d G & =\int_{G}\left[\left(\hat{y}_{e} \cdot \nabla\right) \hat{\varphi}+(\hat{\varphi} \cdot \nabla) \hat{y}_{e}\right] \cdot \psi d G \\
& =\int_{\Omega}\left[\left(y_{e} \cdot \nabla\right) \varphi+(\varphi \cdot \nabla) y_{e}\right] \cdot \psi d \Omega=\int_{\Omega} L_{e}(\varphi) \cdot \psi d \Omega .
\end{aligned}
$$

Then (7.8), (7.9), and (7.1) establish (7.3), that is, (7.2).

STEP 2. In a similar vein, we obtain in $G$,

$$
\operatorname{div} \hat{\varphi}=\left\{\begin{array}{ll}
\operatorname{div} \varphi=0 & \text { in } \Omega ; \\
0 & \text { in } \omega ;
\end{array} \quad \int_{G}(\operatorname{div} \hat{\varphi}) \psi d G=\int_{\Omega}(\operatorname{div} \varphi) \psi d \Omega,\right.
$$

for all $\psi \in C_{0}^{\infty}(G)$; and moreover,

$$
\hat{\varphi} \in\left(H^{2}(G)\right)^{d} ; \quad \hat{p} \in H^{1}(G) .
$$

STEP 3. Thus, the extended triple $\left\{\hat{\varphi}, \hat{p}, \hat{y}_{e}\right\}$ satisfies in $G$ the following Oseen problem:

$$
\begin{cases}(-\Delta) \hat{\varphi}+L_{e}(\hat{\varphi})+\nabla \hat{p}=\lambda \hat{\varphi} & \text { in } G ; \\ \operatorname{div} \hat{\varphi}=0 & \text { in } G ; \\ \left.\hat{\varphi}\right|_{\Gamma_{1}}=0 ; \quad \hat{\varphi} \equiv 0 & \text { in } \omega .\end{cases}
$$


We can now invoke the unique continuation theorem for the Oseen problem $(7.12 \mathrm{a}-\mathrm{c})$ where $\hat{\varphi} \equiv 0$ in the interior subdomain $\omega$ and conclude that

(7.13) $\hat{\varphi} \equiv 0$ in $G ; \quad \hat{p} \equiv$ const in $G ; \quad$ so $\quad \varphi \equiv 0$ in $\Omega ; \quad p \equiv$ const in $\Omega$ (see [F, Section 4], [B-T, Lemma 3.7], and [T.5] for the Oseen problem). References [F-L.1], [F-L.2] give a similar unique continuation result for the Stokes problem. Thus Theorem 3.2 is proved.

\section{II.8. Second proof of Theorem 3.1: Reduction to a biharmonic} equation for $d=2$. The present analysis applies for $d=2$. In this case, it is well known [Te, p. 36] that the Stokes equation can be converted to a biharmonic equation. Presently, we shall reduce (when $d=2$ ) the overdetermined Oseen problem in the unknown $\varphi(x, y)=\left[\varphi_{1}(x, y), \varphi_{2}(x, y)\right]$ :

$$
\begin{cases}(-\Delta) \varphi+L_{e}(\varphi)+\nabla p=\lambda \varphi & \text { in } \Omega \\ \operatorname{div} \varphi \equiv 0 & \text { in } \Omega ; \\ \left.\varphi\right|_{\Gamma} \equiv 0 ;\left.\frac{\partial \varphi}{\partial \nu}\right|_{\Gamma} \equiv 0 & \text { in } \Gamma ; \\ L_{e}(\varphi)=\left(y_{e} \cdot \nabla\right) \varphi+(\varphi \cdot \nabla) y_{e}\end{cases}
$$

to an over-determined biharmonic problem. To this end, define the function

$$
\varrho(x, y) \equiv-\int^{x} \varphi_{2}(\xi, y) d \xi
$$

identified up to a constant, so that

$$
\left\{\begin{array}{l}
\varrho_{x}(x, y)=-\varphi_{2}(x, y) \\
\varrho_{y}(x, y)=-\int^{x} \varphi_{2 y}(\xi, y) d \xi=\int^{x} \varphi_{1 x}(\xi, y) d \xi=\varphi_{1}(x, y),
\end{array}\right.
$$

recalling $\varphi_{1 x}+\varphi_{2 y} \equiv 0$ in $\Omega$, by (8.1b). Thus, [Te, p. 36. Eqn. 2.58]

$$
\varphi=\left[\varphi_{1}, \varphi_{2}\right]=\left[\varrho_{y},-\varrho_{x}\right] .
$$

Proposition 8.1. The change of variable (8.3)-(8.5) transforms the over-determined Oseen problem $(8.1 \mathrm{a}-\mathrm{c})$ in the variables $\{\varphi(x, y), p(x, y)\}$ into the following over-determined biharmonic problem in the variable $\varrho(x, y)$, with three homogeneous boundary conditions:

$$
\left\{\begin{array}{l}
\Delta^{2} \varrho+\lambda \Delta \varrho+\left(y_{e} \cdot \nabla\right)\left[\begin{array}{r}
\varrho_{y} \\
-\varrho_{x}
\end{array}\right]+\left(\left[\begin{array}{r}
\varrho_{y} \\
-\varrho_{x}
\end{array}\right] \cdot \nabla\right) y_{e}=0 \text { in } \Omega ; \\
\left.\varrho\right|_{\Gamma} \equiv 0 ;\left.\quad \frac{\partial \varrho}{\partial \nu}\right|_{\Gamma} \equiv 0,\left.\Delta \varrho\right|_{\Gamma} \equiv 0 .
\end{array}\right.
$$

Proof. First, for the regular Stokes problem, one obtains the first two terms in (8.6a), as well as the first two boundary conditions [Te, p. 36]. For 
short (for $\left.y_{e}=0\right)$ :

$$
\left\{\begin{array}{l}
(-\Delta) \varphi_{1}+p_{x}=\lambda \varphi_{1} \partial_{y} \Rightarrow(-\Delta) \varphi_{1 y}+p_{x y}=\lambda \varphi_{1 y}, \\
(-\Delta) \varphi_{2}+p_{y}=\lambda \varphi_{2} \partial_{x} \Rightarrow(-\Delta) \varphi_{2 x}+p_{y x}=\lambda \varphi_{2 x}
\end{array}\right.
$$

and subtracting (8.7b) from (8.7a) yields

$$
(-\Delta)\left(\varphi_{1 y}-\varphi_{2 x}\right)=\lambda\left(\varphi_{1 y}-\varphi_{2 x}\right), \quad \text { or } \quad \Delta^{2} \varrho+\lambda \Delta \varrho=0,
$$

via (8.5). Next, the Oseen term $L_{e}(\varphi)$ in (8.2) produces the additional term noted in (8.6a). As to the boundary conditions, $\left.\varphi\right|_{\Gamma}=0$ in (8.1c) yields $\left.\varrho_{x}\right|_{\Gamma}=\left.\varrho_{y}\right|_{\Gamma}=0$ via (8.5), hence [L-T.5, p. 299] $\left.\frac{\partial \varrho}{\partial \nu}\right|_{\Gamma}=\varrho_{x} \nu_{1}+\left.\varrho_{y} \nu_{2}\right|_{\Gamma}=0$, and $\left.\frac{\partial \varrho}{\partial \tau}\right|_{\Gamma}=0$, or $\left.\varrho\right|_{\Gamma}=$ const, and we can take $\left.\varrho\right|_{\Gamma}=0$.

Moreover, $\left.\frac{\partial \varphi}{\partial \nu}\right|_{\Gamma}=0$ implies $\left.\frac{\partial \varrho_{y}}{\partial \nu}\right|_{\Gamma}=0$ and $\left.\frac{\partial \varrho_{x}}{\partial \nu}\right|_{\Gamma}=0$ via (8.5), hence via [L-T.1, p. 299]:

$$
\left.\varrho_{x x}\right|_{\Gamma}=\left[\nu_{1} \frac{\partial \varrho_{x}}{\partial \nu}-\nu_{2} \frac{\partial \varrho_{x}}{\partial \nu}\right]_{\Gamma}=0 \quad \text { and }\left.\quad \varrho_{y y}\right|_{\Gamma}=\left[\nu_{2} \frac{\partial \varrho_{y}}{\partial \nu}+\nu_{1} \frac{\partial \varrho_{y}}{\partial \nu}\right]_{\Gamma}=0,
$$

and $\left.\Delta \varrho\right|_{\Gamma}=0$.

The Stokes case $y_{e}=0$. In this case, problem (8.1) becomes

$$
\left\{\begin{array}{l}
\Delta^{2} \varrho+\lambda \Delta \varrho=0 \quad \text { in } \Omega \\
\left.\varrho\right|_{\Gamma}=\left.\frac{\partial \varrho}{\partial \nu}\right|_{\Gamma}=\left.\Delta \varrho\right|_{\Gamma}=0 .
\end{array}\right.
$$

Proposition 8.2. Problem (8.10a-b) implies $\varrho \equiv 0$ in $\Omega$. Accordingly, the Stokes problem $(8.1 \mathrm{a}-\mathrm{c})$ (with $\left.y_{e} \equiv 0\right)$ implies (via $\left.(8.5)\right) \varphi \equiv 0$ in $\Omega$ and $p \equiv$ const in $\Omega$.

Proof. Set $y=\Delta \varrho$ in $\Omega$, which along with $\left.\varrho\right|_{\Gamma}=0$ yields $\varrho=A_{D}^{-1} y$, where $A_{D}$ is the Dirichlet Laplacian, a negative self-adjoint operator on $L_{2}(\Omega)$. Then $(8.10 \mathrm{a}-\mathrm{b})$ is rewritten in the $y$-variable as

$$
\Delta y+\lambda y=0 \quad \text { in } \Omega ;\left.\quad y\right|_{\Gamma}=0,\left.\quad \frac{\partial A_{D}^{-1} y}{\partial \nu}\right|_{\Gamma}=0 .
$$

If $\lambda=0$, then the LHS of the equation in (8.11) implies $y=\Delta \varrho \equiv 0$ in $\Omega$.

If $\lambda \neq 0$, the equation in (8.11) plus the first boundary condition can in turn be rewritten as

$$
A_{D} y+\lambda y=0 \quad \text { or } \quad y+\lambda A_{D}^{-1} y=0 .
$$

Applying $\partial_{\nu}$ to $(8.12)$ and using the second boundary condition in (8.11) yields

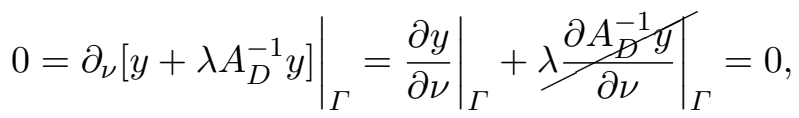


or $\left.\frac{\partial y}{\partial \nu}\right|_{\Gamma}=0$, also when $\lambda \neq 0$. This along with (8.11) yields a standard over-determined problem: $\Delta y=0$ in $\Omega ;\left.y\right|_{\Gamma}=\left.\frac{\partial y}{\partial \nu}\right|_{\Gamma}=0$, and thus it implies again $y \equiv 0$. Thus, for any $\lambda$, we get $y=\Delta \varrho \equiv 0$ in $\Omega$, and $\left.\varrho\right|_{\Gamma}=0$ and hence $\varrho \equiv 0$ in $\Omega$.

The Oseen problem. If $y_{e}$ is small in the $\left(W^{1, \infty}(\Omega)\right)^{d}$-norm $(d=2$ in the present case), a perturbation argument similar to the one in Section II.6 applies and provides the desired conclusion: $(8.1 \mathrm{a}-\mathrm{b}) \Rightarrow \varrho \equiv 0$ in $\Omega$, hence $\varphi \equiv 0$ in $\Omega, p=$ const in $\Omega$.

REMARK 8.1. In 3-d, Isakov [I, p. 62] has the following counterexample. Let $\Omega$ be the unit ball in $\mathbb{R}^{3}$, and let $k_{1}=\pi$ and $k_{2}=2 \pi$. Then there is a solution $u$ which is nonzero a.e. in $\Omega$ of the following fourth-order problem with three boundary conditions:

$$
\left\{\begin{array}{l}
\left(\Delta+k_{1}^{2}\right)\left(\Delta+k_{2}^{2}\right) u=\left\{\Delta^{2}+\left(k_{1}^{2}+k_{2}^{2}\right) \Delta+k_{1}^{2} k_{2}^{2}\right\} u=0 \quad \text { in } \Omega \\
\left.u\right|_{\Gamma}=\left.\frac{\partial u}{\partial \nu}\right|_{\Gamma}=\left.\frac{\partial^{2} u}{\partial \nu^{2}}\right|_{\Gamma}=0
\end{array}\right.
$$

[where, with $\left.u\right|_{\Gamma}=\left.\frac{\partial u}{\partial \nu}\right|_{\Gamma}=0$, we have $\left.\Delta u\right|_{\Gamma}=\left.0 \Leftrightarrow \frac{\partial^{2} u}{\partial \nu^{2}}\right|_{p}=0$.]

Acknowledgements. This research was partially supported by the National Science Foundation under grant DMS-0104305.

The author wishes to thank: George Avalos (Univ. of Nebraska) for reading the first draft of this paper in March 2008; Andrei Fursikov (Moscow State Univ.), Paolo Galdi (Univ. of Pittsburgh), Walter Littman and Vladimir Sverak (Univ. of Minnesota), Victor Isakov (Wichita State University), Igor Kukavica (Univ. of Southern California), J.-P. Raymond (Univ. of Paul Sabatier, Toulouse), Jan Sokołowski (Univ. of Nancy), for sharing information during March-April 2008 on the continuation problem from the boundary of Part II.

\section{References}

[A-T] G. Avalos and R. Triggiani, Boundary feedback stabilization of a coupled parabolic-hyperbolic Stokes-Lamé PDE system, preprint.

[B-L-T.1] V. Barbu, I. Lasiecka, and R. Triggiani, Boundary stabilization of NavierStokes equations, Mem. Amer. Math. Soc. 181 (2006), no. 852.

[B-L-T.2] - - - - , Abstract settings for tangential boundary stabilization of NavierStokes equations by high-and low-gain feedback controllers, Nonlinear Anal. 64 (2006), 2705-2746.

[B-L-T.3] - - - - , Local exponential stabilization strategies of the Navier-Stokes equations, $d=2,3$, via feedback stabilization of its linearization, in: Optimal Control of Coupled Systems of Partial Differential Equations, Int. Ser. Numer. Math. 155, Birkhäuser, 2007, 13-46. 
[B-T] V. Barbu and R. Triggiani, Internal stabilization of Navier-Stokes equations with finitely many controllers, Indiana Univ. Math. J. 53 (2004), 1443-1494.

[B-J-S] L. Bers, F. John, and M. Schechter, Partial Differential Equations, Interscience, 1964.

[C] T. Carleman, Sur le problème d'unicité pour les systèmes d'équations aux dérivées partielles à deux variables indépendantes, Ark. Mat. 26 (1939), $1-9$.

[C-F] P. Constantin and C. Foias, Navier-Stokes Equations, Univ. of Chicago Press, 1989.

[F-L.1] C. Fabre and G. Lebeau, Prolongement unique des solutions de l'équation de Stokes, Comm. Partial Differential Equations 21 (1996), 573-596.

[F-L.2] - - - Regularité et unicité pour le problème de Stokes, ibid. 27 (2002), 437-475.

[F] A. Fursikov, Stabilization of the 3D Navier-Stokes system by feedback boundary control, Discrete Contin. Dynam. Systems 10 (2004), 289-314.

[H] L. Hörmander, The Analysis of Linear Partial Differential Operators III, Springer, 1985.

[I] V. Isakov, Inverse Problems for Partial Differential Equations, 2nd ed., Springer, 2006.

[Ka] T. Kato, Perturbation Theory for Linear Operators, Springer, 1967.

[Kom] V. Komornik, Exact Controllability and Stabilization-The Multiplier Method, Masson, 1994.

[Kon] V. A. Kondrat'ev, Boundary problems for elliptic equations in domains with conical or angular points, Trans. Moscow Math. Soc. 16 (1967), 227-313.

[L-T.1] I. Lasiecka and R. Triggiani, Structural assignment of Neumann boundary feedback parabolic equations: The case of trace in the feedback loop, Ann. Mat. Pura Appl. (4) 32 (1982), 131-175.

[L-T.2] -, - Stabilization of Neumann boundary feedback parabolic equations: The case of trace in the feedback loop, Appl. Math. Optim. 10 (1983), 307-350.

[L-T.3] -, - Stabilization and structural assignment of Dirichlet boundary feedback parabolic equations, SIAM J. Control Optim. 21 (1983), 766-803.

[L-T.4] - - - Feedback semigroups and cosine operators for boundary feedback parabolic and hyperbolic equations, J. Differential Equations 47 (1983), 246-272.

[L-T.5] - - - Control Theory for Partial Differential Equations: Continuous and Approximation Theories, Vol. 1, Encyclopedia Math. Appl. 74, Cambridge Univ. Press, 2000.

[L-T.6] -, 一, Uniform stabilization with arbitrary decay rates of the Oseen equation by finite-dimensional tangential boundary and localized interior controls, preprint, 2008.

[M] C. Miranda, Partial Differential Equations of Elliptic Type, Springer, 1970.

[R] J.-P. Raymond, Feedback boundary stabilization of the two-dimensional Navier-Stokes equations, SIAM J. Control Optim. 45 (2006), 790-828.

[S-W] G. Schmidt and N. Weck, On the boundary behavior of solutions to parabolic equations, ibid. 16 (1978), 533-598.

[S-Z] J. Sokołowski and J. P. Zolésio, Introduction to Shape Optimization. Shape Sensitivity Analysis, Springer Ser. Comput. Math. 16, Springer, 1992.

[Te] R. Temam, Navier-Stokes Equations, Stud. Math. Appl. 2, North-Holland, Amsterdam, 1979.

[T.1] R. Triggiani, On Nambu's boundary stabilizability problem for diffusion processes, J. Differential Equations 33 (1979), 189-200. 
[T.2] R. Triggiani, Well-posedness and regularity of boundary feedback parabolic systems, ibid. 36 (1980), 347-362.

[T.3] - Boundary feedback stabilizability of parabolic equations, Appl. Math. Optim. 6 (1980), 201-220.

[T.4] -, Unique continuation of boundary over-determined Stokes and Oseen problems, Discrete Contin. Dynam. Systems, to appear.

[T.5] - Unique continuation from an arbitrary interior subdomain of the variable coefficient Oseen equation, preprint, 2008.

Department of Mathematics

University of Virginia

Charlottesville, VA 22903, U.S.A.

E-mail: rt7u@virginia.edu

Received on 5.6.2007;

revised version on 30.10.2008 